\title{
Internet Appendix to Do the Rich Get Richer in the Stock Market? Evidence from India
}

\author{
John Y. Campbell, Tarun Ramadorai, and Benjamin Ranish ${ }^{1}$
}

First draft: March 2018

This draft: July 2018

\footnotetext{
${ }^{1}$ Campbell: Department of Economics, Littauer Center, Harvard University, Cambridge MA 02138, USA, and NBER. john_campbell@harvard.edu. Ramadorai: Imperial College London, London SW7 2AZ, UK. t.ramadorai@imperial.ac.uk. Ranish: Federal Reserve Board, 20th \& C Streets NW, Washington DC 20551, USA. ben.ranish@frb.gov. We gratefully acknowledge NSDL and SEBI for providing us with access to the data, the Sloan Foundation for financial support, and Imperial College for the use of computing facilities. The opinions expressed in this paper are those of the authors and do not necessarily reflect the views of the Board of Governors or other employees of the Federal Reserve System.
} 


\section{Contents}

1 Data Construction $\quad 2$

1.1 Stock-Level Data . . . . . . . . . . . . . . . . . . . . 2

1.2 NSDL Account Data . . . . . . . . . . . . . . . . . . . . . 2

2 Additional Exercises, Explanations, and Extensions of Results 3

2.1 Equities and Other Assets of Indian Households . . . . . . . . . . . . . . . 3

2.2 Indirect Individual Equity Ownership in India . . . . . . . . . . . . . . . . 4

2.3 Comparison of Alternative Inequality Measures . . . . . . . . . . . . . . . 4

2.4 Contributors to Inequality Growth at Different Points in Time . . . . . . . . 4

3 Robustness Exercises $\quad 5$

3.1 What are Risk and Returns Across the Largest Accounts? . . . . . . . . . . 5

3.2 Do Results Reflect Account Age or Experience Effects? . . . . . . . . . . . . 5

3.3 Do Results Reflect Heterogeneous Skill? . . . . . . . . . . . . . . . . 5

3.4 Do Results Reflect Measurement Issues Affecting Micro-Cap Stocks? . . . . . 6

3.5 Are Results Driven by Unusual Accounts? . . . . . . . . . . . . . . . . 6

3.6 Role of Account Exit Due to Return Availability versus Exit Due to Sales . . 7

$\begin{array}{llr}4 & \text { Tables and Figures } & 7\end{array}$ 


\section{Data Construction}

\subsection{Stock-Level Data}

We collect stock-level data on monthly total returns, market capitalization, and book value from three sources: Compustat Global, Datastream, and Prowess. Prowess reports data from both of India's major stock exchanges, the Bombay and National Stock Exchanges (BSE and NSE). In addition, monthly price returns can be inferred from the month-end holding values and quantities in the NSDL database. We link the datasets by ISIN. ${ }^{2}$

To verify reliability of returns data, we compare total returns from the available data sources, computing the absolute differences in returns series across sources. For each stockmonth, we use returns from one of the datasets for which returns match another dataset most closely, where the source from amongst those datasets is selected in the following order of priority: Compustat Global, Prowess NSE, then Prowess BSE. If returns are available from only one source, or the difference(s) between the multiple sources all exceed $5 \%$ then we compare price returns from each source with price returns from NSDL. We then use total returns from the source for which price returns most closely match NSDL price returns, provided the discrepancy is less than $5 \%$.

After computing total returns, we drop extended zero-return periods which appear for non-traded securities. We also drop first (partial) month returns on IPOs and re-listings, which are reported inconsistently. For the 25 highest and 25 lowest remaining total monthly returns, we use internet sources such as Moneycontrol and Economic Times to confirm that the returns are valid. We also use internet sources to look up and confirm returns for stockmonths where returns are missing and the stock comprises at least one percent of stock holdings for the representative individual investor for either the previous or current month.

We follow a similar data verification procedure for market capitalization and book value, confirming that the values used are within $5 \%$ of that reported by another source. Where market capitalization cannot be determined for a given month, we extrapolate it from the previous month using price returns. Where book value is unknown, we extrapolate it forward using the most recent observation over the past year.

\subsection{NSDL Account Data}

Our NSDL data are monthly frequency, and include the trades and holdings of all NSDL accounts from February 2002 through May 2011. ${ }^{3}$ Table A1 shows the number of unique

\footnotetext{
${ }^{2}$ Around dematerialisation, securities' ISINs change, with some data linked to pre-dematerialisation ISINs and other data linked to post-dematerialisation ISINs. We use a matching routine and manual inspection to match the ISINs that represent the same security.

${ }^{3}$ These data are collected with the approval of India's securities markets regulator, SEBI.
} 
securities held in NSDL accounts, which ranges from a bit over 8,000 in 2002 to almost 22,000 in 2011 . While the number of unique equities is only $1 / 3$ to $1 / 2$ as large, equities are by far the most commonly held, and comprise the bulk of NSDL holdings.

Table A1 shows that equity holdings in NSDL account for a growing share of India's market capitalization, from about 45\% in 2002 and approaching 70\% in 2011. Most of the remaining Indian equities are held in accounts in Central Depository Services Limited (CDSL). The two depositories combined share of Indian equity holdings is now close to $100 \%$ of the market. While the vast majority of accounts are held by individuals, these accounts represent only about $20 \%$ of NSDL account holdings by value in 2002. The individual account share declines gradually to about $10 \%$ at the end of our data sample as institutional investors enter the market. ${ }^{4}$ Mutual funds' share of equity holdings remains small throughout the sample, and never represents more than $5 \%$ of aggregate NSDL stock holdings.

In this paper, we restrict our attention to the equity holdings of individual Indian resident accounts, discarding the relatively small number of such accounts that never hold equities. In addition, we aggregate together individual accounts sharing the same permanent account number (PAN), which is unique to an individual. Our sample of 200,000 accounts is drawn from this set of roughly 11.6 million individual PAN-aggregated accounts.

\section{Additional Exercises, Explanations, and Extensions of Results}

\subsection{Equities and Other Assets of Indian Households}

We use the All India Debt and Investment Survey (conducted by the Reserve Bank of India in the 2012) to show that the value of households' equities are significantly related to the amount of other household assets. Specifically, Table A2 shows the output from least squares regressions of household assets on the value of household equity holdings. To deal with the extreme right skewness of household wealth, Panel A provides results using log assets and Panel B provides results using variables converted to rank percentile.

The results in Table A2 suggest that, on average, a household with one percent more stock holdings has about 0.15 percent greater total assets, and a household ranked one percentile higher in stock holdings is ranked about 0.3 percentiles higher in total assets.

\footnotetext{
${ }^{4}$ We classify any account which holds greater than $5 \%$ of a stock with market capitalization above 500 million Rs (approximately $\$ 10$ million) as a "beneficial owner" account if that account would otherwise be classied as a trust, body corporate,or individual account. This separates accounts with significant control rights from standard investment accounts. Otherwise our account classifications are many-to-one mappings based on the detailed investor types we observe.
} 


\subsection{Indirect Individual Equity Ownership in India}

To obtain estimates of individuals' indirect Indian equity holdings, we use data from the Association of Mutual Funds of India on the aggregate value of mutual funds and unit trusts, and data from the Insurance Regulatory and Development Authority on the aggregate value fo unit-linked insurance plan premiums. We assume these vehicles account for all indirect ownership of Indian equities. We classify "growth" (or "equity") and "equity linked savings schemes" as fully invested in stock, and funds classified as "balanced" as well as unit-linked insurance as invested half in stocks. Of these categories, "growth" /" equity" funds is by far the largest. We assume that individuals own a similar fraction of equity mutual funds and non-equity mutual funds, and obtain this fraction (which averages around 40\%) from SEBI reports. ${ }^{5}$

Next, we extrapolate the value of individuals' direct ownership of stock as $5 / 3$ of that held by individual accounts registered in NSDL, based on NSDL having an approximately $60 \%$ share of all such accounts. Our estimate of indirect holdings varies between $6 \%$ and $19 \%$ of total household equity holdings over 2002 through 2011. In addition, a 2009 SEBI survey found that about $65 \%$ of Indian households owning individual stocks did not own any bonds or mutual funds.

\subsection{Comparison of Alternative Inequality Measures}

We have characterized the entire distribution of log account size using the cross-sectional variance for expositional ease. However, Figure A1 shows that both the Gini coefficient and the share of market capitalization held by the top $5 \%$ of accounts have trended upwards in a similar manner. Since these alternative inequality measures are far more sensitive to the extreme right tail of the wealth distribution, this suggests that our analysis may still be relevant even if one is particularly concerned with concentration of wealth at the very top.

In addition, the black curves in Figure A1 compare the cross-sectional variance of log account size in our sample with that of the full set of 11.6 million individual accounts. Given that our sample is drawn at random, the two series are unsurprisingly almost identical.

\subsection{Contributors to Inequality Growth at Different Points in Time}

Table 4 of our paper presents the time-series average of the several components of the growth in variance of log account value. Did these components' contributions vary significantly over time? Figure A2 suggests that heterogeneous returns make the most stable contribution to the variance of $\log$ account size. In contrast, flows into and out of accounts primarily

\footnotetext{
${ }^{5}$ We use data from 2003 for 2004 through 2009, as we are unable to locate the figure for these intermediate dates.
} 
increased this variance in the beginning of the sample, whereas account exits decreased inequality in the beginning of the sample and increased inequality primarily in 2007 and 2008.

\section{Robustness Exercises}

\subsection{What are Risk and Returns Across the Largest Accounts?}

Table A3 replicates Table 2 (Panel A) and Table 3 from our paper using size-sorted deciles within the largest $5 \%$ of accounts. ${ }^{6}$ Return and risk are similar across the largest $5 \%$ of accounts. However, there is some evidence that the very largest accounts (top $0.5 \%$ of all accounts) have four-factor alpha.

\subsection{Do Results Reflect Account Age or Experience Effects?}

We have focused on account size rather than account age as in our earlier work. Could we be confusing account size effects with account age effects, for example a tendency for investors to diversify and add to their accounts over time? To address this concern, in Table A4 we replicate Table 2 (Panel A) and Table 3 using cohort-balanced size deciles. Accounts in these deciles are weighted such that at each point in time, the distribution of cohorts (age) within each decile equals the distribution of cohorts across all size deciles. ${ }^{7}$ Results across cohort-balanced size deciles are very similar to our primary analysis, suggesting our results are not driven by account age effects.

\subsection{Do Results Reflect Heterogeneous Skill?}

We have used accounts' market values to measure their size even though returns affect subsequent market values. Could it be that large accounts earn higher average log returns because there is persistent cross-sectional variation in investment skill, and skillful investors have accounts that become large? To address this, we construct two alternative measures of account size that remove the effect of returns on size. The first of these, which we call "book value of account" by analogy with the corporate accounting concept, is equal to the value of each account under the counterfactual assumption that the account earns an Indian money market rate while having the same rupee inflows and the same proportional outflows that actually occurred. The rupee inflows are assumed to be the same as the counterfactual

\footnotetext{
${ }^{6}$ In order to have a sufficient sample size, we draw a new random sample of 200,000 accounts from the set of accounts that are in the top $5 \%$ of stock holding value for at least one month. We only include in the analysis those months immediately following a size ranking in the top $5 \%$.

${ }^{7}$ Accounts in cohort-balanced deciles are equally weighted within each month-decile-cohort group.
} 
assumes the same amount of outside money is available for stock investment. As a simpler alternative, we also define "alternative book value of account" as the cumulated sum of net inflows. A difficulty with this alternative measure is that it need not be positive, and in particular will be negative for investors who live off their capital income in steady state.

Table A5 provides summary statistics for the log of these alternative size measures alongside log account value. The size measures have correlations exceeding 0.9 with each other, and the measures have similar correlations with account growth and returns. ${ }^{8}$ In Table A6 we replicate Table 2 (Panel A) and Table 3 using book value deciles instead of account size. In Table A7, we do the same for deciles based on alternative book value. Risk and return relationships are similar across all three size measures.

\subsection{Do Results Reflect Measurement Issues Affecting Micro-Cap Stocks?}

Small Indian investors often hold micro-cap stocks as documented in Panel B of Table 2. We have included micro-cap stock returns when we can verify their returns from two data sources, but one might be concerned that the average simple returns on these stocks are biased upwards if there is survivorship bias for micro-cap stocks in the underlying data sources, or if these stocks are so illiquid that their returns are strongly negatively autocorrelated (Blume and Stambaugh 1983).

In Table A8 we replicate Table 2 (Panel A) and Table 3 after removing returns for stocks with aggregate holdings in NDSL below 500 million Rs (approximately $\$ 10$ million) at the end of the prior month. This modification reduces both excess returns and excess return volatility, particularly for accounts in the smallest decile. However, risk and return trends across size deciles are qualitatively unchanged, with mean log returns increasing with account size.

\subsection{Are Results Driven by Unusual Accounts?}

Some portfolios may not accurately represent the investor's long-run investment approach. An account that was recently opened might be actively evolving, with the investor steadily adding funds and positions as they figure out a strategy. Investors who won shares through participation in a major IPO, and who have little or no other equity investment, might be only incidental participants in the stock market. Investors who exit and enter the stock market periodically may not be using their stock investments primarily to meet long-run savings objectives. If these investor types disproportionately have small account values, then there may be concern that increasing account value inequality is significantly driven not by

\footnotetext{
${ }^{8}$ These correlations exclude observations where book value is negative.
} 
differences in the portfolios of "normal" small and large investors, but by the particular objectives of unusual investor types who happen to be small equity investors.

To address this concern, we re-run the decomposition of the change in variance of log account value (Table 4) using data sets with potentially "unusual" investors filtered out. Table A9 provides this replication excluding "IPO investors." IPO investor accounts are the $27.5 \%$ of accounts which, in at least one month, have at least $80 \%$ of the account equity value invested in stocks within three months of their IPO. Table A10 replicates Table 4 while excluding the first 12 months of each account's equity investing history. Table A11 replicates Table 4 while excluding accounts that exit the stock market for a period of more than one consecutive month during our sample period. ${ }^{9}$

These tables show that the overall growth of the variance of log account value is similar in these filtered data sets to that of the full data set. Furthermore, a similar (0.0144 to 0.0176) amount of growth is due to the role of account returns across the three datasets. Even excluding new accounts, IPO accounts, and potential short-term investors, small accounts are far less diversified with lower log returns.

\subsection{Role of Account Exit Due to Return Availability versus Exit Due to Sales}

In Panel A of Table 4, we lump together two types of account entry and exit. Accounts can enter either because they start to hold stocks, or because at least one of the stocks they hold starts to have a measured return. Similarly, accounts can exit either because they cease to hold stocks, or because none of the stocks they hold continue to have measured returns. To what extent do these two types of entry and exit contribute differently? Table A12 shows that entry and exit driven by stockholdings increases account value inequality, while entry and exit driven by the availability of returns data decreases it. The first effect is substantial at $40 \%$ of the observed increase in the cross-sectional variance of log account size, but the second effect is also substantial at $-41 \%$, and the sum of the two effects is the modest $-1 \%$ contribution of account entry and exit reported in Panel A of Table 4.

\section{Tables and Figures}

\footnotetext{
${ }^{9}$ Some long-run investors may not be invested in stocks at month-end if they have not yet had time to reinvest the proceeds of stock sales near the month's end. Therefore, we do not exclude accounts that appear to have exited the stock market for only a single month at a time.
} 
Figure A1: Evolution of Inequality Measures

Top 5\% Share Increased by 15\% for Display Purposes

—Gini —Top 5\% Share (+15\%) - Var Log Account Value -- Var Log Account Value (Sample)

0.94

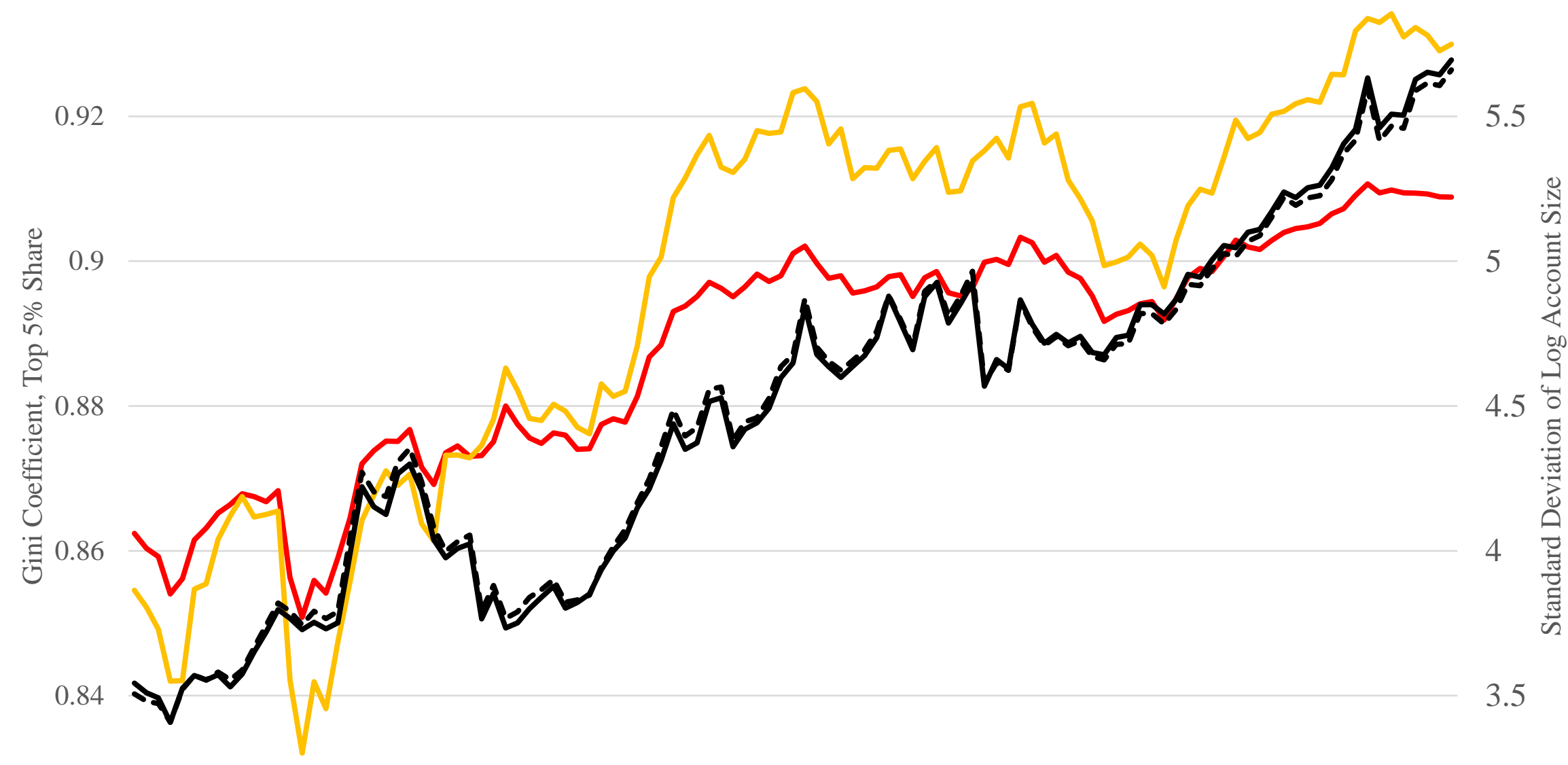

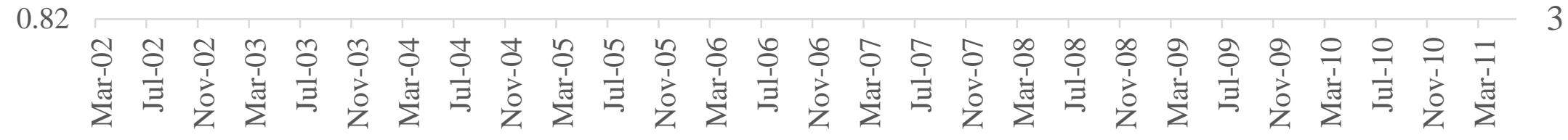


Figure A2: Decomposition of Cumulative Change in Account Size Variance

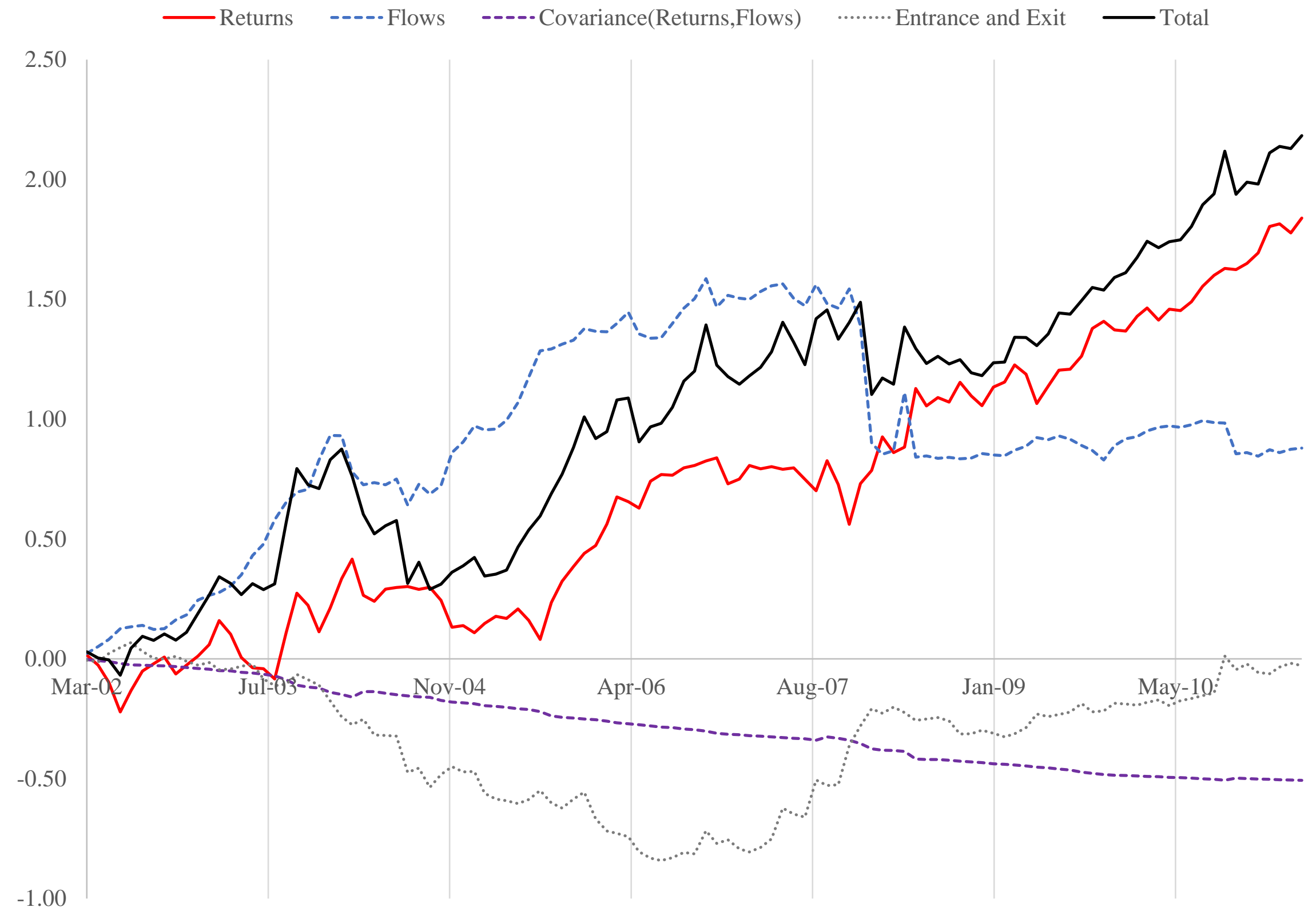


Table A1: Summary Statistics for NSDL Database

The percentages below are computed for each monthly cross-section, and the average of these monthly percentages within each year appear in the table. The number of unique securities and equities are determined by the average number of unique ISIN appearing in the NSDL database in each month in the given year (from Feb 2002 through May 2011). Individual accounts exclude "beneficial owners" (those holding more than 5\% of the market capitalization of a stock at some point in time). BSE market capitalization (from the World Federation of Exchanges), is from the end of each year and represents the market capitalization of all equities listed on the BSE, representing the vast majority of Indian equities. December 2002 market capitalization is imputed from January 2003 returns and month-end capitalization.

\begin{tabular}{|c|c|c|c|c|c|c|}
\hline & $\begin{array}{c}\text { Number of Unique } \\
\text { Securities }\end{array}$ & $\begin{array}{l}\text { Number of Unique } \\
\text { (Indian) Equities }\end{array}$ & $\begin{array}{c}\text { Market Capitalization } \\
\text { of BSE (Billions of } \\
\text { US\$) }\end{array}$ & $\begin{array}{l}\text { \% of Indian Equity } \\
\text { Market Capitalization } \\
\text { in NSDL Accounts }\end{array}$ & $\begin{array}{c}\% \text { of NSDL Equity } \\
\text { Value in Individual } \\
\text { Accounts }\end{array}$ & $\begin{array}{c}\% \text { of NSDL Equity } \\
\text { Value in Mutual Funds }\end{array}$ \\
\hline 2002 & 8,245 & 3,788 & $\$ 117.4$ & $44.33 \%$ & $19.03 \%$ & $2.11 \%$ \\
\hline 2003 & 11,054 & 4,137 & $\$ 279.1$ & $45.25 \%$ & $18.49 \%$ & $3.50 \%$ \\
\hline 2004 & 12,264 & 4,406 & $\$ 387.9$ & $51.19 \%$ & $16.89 \%$ & $3.51 \%$ \\
\hline 2005 & 13,487 & 4,711 & $\$ 553.1$ & $58.33 \%$ & $15.41 \%$ & $3.73 \%$ \\
\hline 2006 & 15,279 & 5,016 & $\$ 818.9$ & $63.90 \%$ & $14.58 \%$ & $4.72 \%$ \\
\hline 2007 & 17,091 & 5,364 & $\$ 1,819.1$ & $66.98 \%$ & $12.66 \%$ & $4.55 \%$ \\
\hline 2008 & 17,511 & 5,829 & $\$ 647.2$ & $65.67 \%$ & $11.71 \%$ & $4.46 \%$ \\
\hline 2009 & 17,458 & 6,239 & $\$ 1,306.5$ & $64.78 \%$ & $10.98 \%$ & $4.56 \%$ \\
\hline 2010 & 19,458 & 6,711 & $\$ 1,631.8$ & $68.02 \%$ & $10.60 \%$ & $4.34 \%$ \\
\hline 2011 & 21,783 & 7,151 & $\$ 1,007.2$ & $67.77 \%$ & $10.10 \%$ & $3.98 \%$ \\
\hline
\end{tabular}




\section{Table A2: Regressions of Total Assets on Stock Holdings}

The table below summarizes regressions of Indian household assets (with or without stocks) on household stock holdings for all households with non-zero stock holdings. Panel A presents results using log assets and stock holdings, while Panel B performs the same regression using percentile rank transformed variables. Data come from the All India Debt and Investment Survey conducted by the Reserve Bank of India in 2012. Sample weights are accounted for in the regressions, and robust standard errors are reported in parentheses.

Panel A: Log Assets and Stock Holdings

\begin{tabular}{lcc} 
& All Assets & All Assets Except Stocks \\
\hline \multirow{2}{*}{ Constant } & {$[\mathrm{i}]$} & {$[\mathrm{ii}]$} \\
& 13.25 & 13.30 \\
Stock Holdings & $(0.13)$ & $(0.13)$ \\
& 0.16 & 0.15 \\
\hline R-Squared & $(0.02)$ & $(0.02)$ \\
Observations & 0.09 & 0.08 \\
& 3,729 & 3,729
\end{tabular}

Panel B: Percentile Rank Assets and Stock Holdings

\begin{tabular}{lcc} 
& All Assets & All Assets Except Stocks \\
\hline \multirow{3}{*}{ Constant } & {$[\mathrm{i}]$} & {$[\mathrm{ii}]$} \\
& 35.43 & 35.97 \\
Stock Holdings & $(1.65)$ & $(1.64)$ \\
& 0.29 & 0.28 \\
R-Squared & $(0.03)$ & $(0.03)$ \\
Observations & 0.08 & 0.08 \\
& 3,729 & 3,729
\end{tabular}


Table A3: Risk and Return by Account Value Deciles Within Largest 5\% of Accounts

Panel A replicates Table 2 for account-value sorted deciles within the largest 5\% of accounts, i.e. decile one spans the 95th through 95.5th percentile.

Panel B does the same for Table 3. Standard errors are reported in parentheses.

Panel A: Return Factor Loadings

Excess Return

\begin{tabular}{|c|c|c|c|c|c|c|c|c|c|c|}
\hline Smallest & 2 & 3 & 4 & 5 & 6 & 7 & 8 & & Largest & Smallest \\
\hline $\begin{array}{c}1.69 \% \\
(0.82 \%)\end{array}$ & $\begin{array}{c}1.72 \% \\
(0.82 \%)\end{array}$ & $\begin{array}{c}1.70 \% \\
(0.81 \%)\end{array}$ & $\begin{array}{c}1.69 \% \\
(0.81 \%)\end{array}$ & $\begin{array}{c}1.69 \% \\
(0.81 \%)\end{array}$ & $\begin{array}{c}1.71 \% \\
(0.80 \%)\end{array}$ & $\begin{array}{c}1.70 \% \\
(0.80 \%)\end{array}$ & $\begin{array}{c}1.70 \% \\
(0.79 \%)\end{array}$ & $\begin{array}{c}1.70 \% \\
(0.78 \%)\end{array}$ & $\begin{array}{c}1.74 \% \\
(0.78 \%)\end{array}$ & $\begin{array}{c}0.05 \% \\
(0.11 \%)\end{array}$ \\
\hline $\begin{array}{c}0.38 \% \\
(0.37 \%)\end{array}$ & $\begin{array}{c}0.41 \% \\
(0.36 \%)\end{array}$ & $\begin{array}{c}0.42 \% \\
(0.35 \%)\end{array}$ & $\begin{array}{c}0.42 \% \\
(0.36 \%)\end{array}$ & $\begin{array}{c}0.42 \% \\
(0.35 \%)\end{array}$ & $\begin{array}{c}0.46 \% \\
(0.36 \%)\end{array}$ & $\begin{array}{c}0.47 \% \\
(0.35 \%)\end{array}$ & $\begin{array}{c}0.50 \% \\
(0.35 \%)\end{array}$ & $\begin{array}{c}0.56 \% \\
(0.36 \%)\end{array}$ & $\begin{array}{c}0.64 \% \\
(0.37 \%)\end{array}$ & $\begin{array}{c}0.25 \% \\
(0.11 \%)\end{array}$ \\
\hline $\begin{array}{c}1.01 \\
(0.02)\end{array}$ & $\begin{array}{c}1.00 \\
(0.02)\end{array}$ & $\begin{array}{c}1.00 \\
(0.02)\end{array}$ & $\begin{array}{c}1.00 \\
(0.02)\end{array}$ & $\begin{array}{c}1.00 \\
(0.02)\end{array}$ & $\begin{array}{c}0.99 \\
(0.02)\end{array}$ & $\begin{array}{c}0.99 \\
(0.02)\end{array}$ & $\begin{array}{c}0.98 \\
(0.02)\end{array}$ & $\begin{array}{c}0.98 \\
(0.02)\end{array}$ & $\begin{array}{c}0.97 \\
(0.02)\end{array}$ & $\begin{array}{l}-0.03 \\
(0.01)\end{array}$ \\
\hline $\begin{array}{c}0.06 \\
(0.02)\end{array}$ & $\begin{array}{c}0.06 \\
(0.02)\end{array}$ & $\begin{array}{c}0.06 \\
(0.02)\end{array}$ & $\begin{array}{c}0.06 \\
(0.02)\end{array}$ & $\begin{array}{c}0.06 \\
(0.02)\end{array}$ & $\begin{array}{c}0.06 \\
(0.02)\end{array}$ & $\begin{array}{c}0.06 \\
(0.02)\end{array}$ & $\begin{array}{c}0.06 \\
(0.02)\end{array}$ & $\begin{array}{c}0.06 \\
(0.02)\end{array}$ & $\begin{array}{c}0.07 \\
(0.02)\end{array}$ & $\begin{array}{c}0.01 \\
(0.01)\end{array}$ \\
\hline $\begin{array}{l}-0.07 \\
(0.10)\end{array}$ & $\begin{array}{l}-0.07 \\
(0.09)\end{array}$ & $\begin{array}{l}-0.08 \\
(0.09)\end{array}$ & $\begin{array}{l}-0.08 \\
(0.09)\end{array}$ & $\begin{array}{l}-0.08 \\
(0.09)\end{array}$ & $\begin{array}{c}-0.09 \\
(0.09)\end{array}$ & $\begin{array}{l}-0.10 \\
(0.09)\end{array}$ & $\begin{array}{c}-0.11 \\
(0.09)\end{array}$ & $\begin{array}{c}-0.13 \\
(0.10)\end{array}$ & $\begin{array}{l}-0.14 \\
(0.10)\end{array}$ & $\begin{array}{l}-0.07 \\
(0.02)\end{array}$ \\
\hline $\begin{array}{l}-0.06 \\
(0.03)\end{array}$ & $\begin{array}{l}-0.07 \\
(0.03)\end{array}$ & $\begin{array}{l}-0.06 \\
(0.03)\end{array}$ & $\begin{array}{l}-0.06 \\
(0.03)\end{array}$ & $\begin{array}{l}-0.05 \\
(0.03)\end{array}$ & $\begin{array}{l}-0.05 \\
(0.03)\end{array}$ & $\begin{array}{c}-0.04 \\
(0.03)\end{array}$ & $\begin{array}{l}-0.03 \\
(0.03)\end{array}$ & $\begin{array}{l}-0.03 \\
(0.03)\end{array}$ & $\begin{array}{l}-0.03 \\
(0.04)\end{array}$ & $\begin{array}{c}0.03 \\
(0.02)\end{array}$ \\
\hline
\end{tabular}

Four-Factor Alpha

Market Beta

Size (SMB)

Value (HML)

Momentum (MOM)

$(0.03)$

$(0.03)$

(0.03)

Account Value Deciles

Largest minus

Realized:

Excess Returns

Smallest $4 \quad 5 \quad 6$

7

8

9 Larges

Smallest

Excess Log Returns

$\begin{array}{llllllllllll}(0.82 \%) & (0.82 \%) & (0.81 \%) & (0.81 \%) & (0.81 \%) & (0.80 \%) & (0.80 \%) & (0.79 \%) & (0.78 \%) & (0.78 \%) & (0.11 \%)\end{array}$

$\begin{array}{lllllllllll}1.10 \% & 1.13 \% & 1.11 \% & 1.11 \% & 1.10 \% & 1.13 \% & 1.12 \% & 1.12 \% & 1.12 \% & 1.09 \% & -0.01 \%\end{array}$

Excess Return Volatility

$\begin{array}{llllllllllll}(0.82 \%) & (0.82 \%) & (0.81 \%) & (0.81 \%) & (0.81 \%) & (0.80 \%) & (0.80 \%) & (0.79 \%) & (0.79 \%) & (0.79 \%) & (0.12 \%)\end{array}$

Sharpe Ratio

$10.9 \% \quad 10.9 \%$

$\begin{array}{llll}(0.8 \%) & (0.8 \%) & (0.8 \%) & (0.8 \%)\end{array}$

$10.8 \% \quad 10.9 \%$

$10.8 \% \quad 10.8 \% \quad 10.8 \% \quad 11.6 \% \quad 0.6 \%$

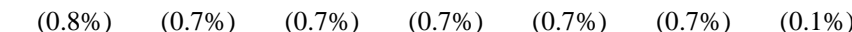

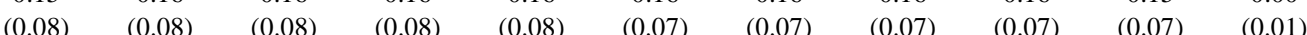

Long Run Global Factor Prices:

Excess Returns

Excess Log Returns

$\begin{array}{lllllllllll}0.86 \% & 0.88 \% & 0.88 \% & 0.89 \% & 0.89 \% & 0.92 \% & 0.94 \% & 0.96 \% & 1.02 \% & 1.09 \% & 0.23 \%\end{array}$

$\begin{array}{lllllllllll}(0.44 \%) & (0.43 \%) & (0.43 \%) & (0.43 \%) & (0.43 \%) & (0.43 \%) & (0.43 \%) & (0.43 \%) & (0.43 \%) & (0.44 \%) & (0.11 \%)\end{array}$

$\begin{array}{lllllllllll}0.26 \% & 0.28 \% & 0.29 \% & 0.31 \% & 0.30 \% & 0.34 \% & 0.35 \% & 0.38 \% & 0.43 \% & 0.43 \% & 0.17 \%\end{array}$

Sharpe Ratio

$\begin{array}{lllllll}(0.45 \%) & (0.44 \%) & (0.44 \%) & (0.44 \%) & (0.43 \%) & (0.44 \%) & (0.4\end{array}$

$\begin{array}{lllll}(0.44 \%) & (0.43 \%) & (0.44 \%) & (0.45 \%) & (0.12 \%)\end{array}$

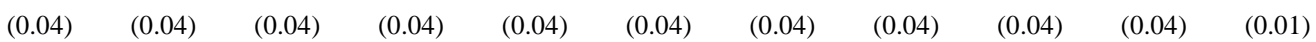

Long Run Global Factor Prices, No Alpha:

Excess Returns

$0.47 \%$

Excess Log Returns

$(0.24 \%) \quad(0.24 \%)$

(0.24\%)

$-0.13 \% \quad-0.13 \%$

(0.24\%)

$(0.04)$

(0.04)

Sharpe Ratio

$\begin{array}{ccccccccccc}0.04 & 0.04 & 0.04 & (0.25 \%) & (0.25 \%) & (0.25 \%) & (0.25 \%) & (0.25 \%) & (0.25 \%) & (0.25 \%) & (0.02 \%) \\ (0.02) & (0.02) & (0.02) & (0.02) & (0.02) & (0.02) & (0.02) & (0.02) & (0.02) & (0.02) & (0.00)\end{array}$


Table A4: Risk and Return by Cohort-Balanced Account Value Deciles

Panel A replicates Table 2 using account-value sorted deciles which are balanced by account age cohort. Specifically, the weight of each age cohort of accounts within each size decile is set equal to the age cohort's share of all accounts in the cross-section. Within each cross-section, accounts within a given cohort and size-decile are equally weighted. Panel B similarly replicates Table 3 using cohort-balanced account-value deciles. Standard errors are reported in parentheses.

Panel A: Return Factor Loadings

Excess Return

$$
\text { ings }
$$

\begin{tabular}{|c|c|c|c|c|c|c|c|c|c|c|c|}
\hline Excess Return & $\begin{array}{c}2.95 \% \\
(1.36 \%)\end{array}$ & $\begin{array}{c}2.03 \% \\
(1.13 \%)\end{array}$ & $\begin{array}{c}2.11 \% \\
(1.03 \%)\end{array}$ & $\begin{array}{c}1.95 \% \\
(0.97 \%)\end{array}$ & $\begin{array}{c}1.87 \% \\
(0.94 \%)\end{array}$ & $\begin{array}{c}1.81 \% \\
(0.93 \%)\end{array}$ & $\begin{array}{c}1.78 \% \\
(0.92 \%)\end{array}$ & $\begin{array}{c}1.75 \% \\
(0.90 \%)\end{array}$ & $\begin{array}{c}1.74 \% \\
(0.88 \%)\end{array}$ & $\begin{array}{c}1.66 \% \\
(0.84 \%)\end{array}$ & $\begin{array}{l}-1.29 \% \\
(0.79 \%)\end{array}$ \\
\hline Four-Factor Alpha & $\begin{array}{l}-0.08 \% \\
(0.70 \%)\end{array}$ & $\begin{array}{l}-0.19 \% \\
(0.63 \%)\end{array}$ & $\begin{array}{c}0.11 \% \\
(0.53 \%)\end{array}$ & $\begin{array}{c}0.17 \% \\
(0.50 \%)\end{array}$ & $\begin{array}{c}0.20 \% \\
(0.46 \%)\end{array}$ & $\begin{array}{c}0.19 \% \\
(0.45 \%)\end{array}$ & $\begin{array}{c}0.18 \% \\
(0.44 \%)\end{array}$ & $\begin{array}{c}0.19 \% \\
(0.41 \%)\end{array}$ & $\begin{array}{c}0.22 \% \\
(0.38 \%)\end{array}$ & $\begin{array}{c}0.32 \% \\
(0.37 \%)\end{array}$ & $\begin{array}{c}0.40 \% \\
(0.43 \%)\end{array}$ \\
\hline Market Beta & $\begin{array}{c}1.20 \\
(0.05)\end{array}$ & $\begin{array}{c}1.16 \\
(0.06)\end{array}$ & $\begin{array}{c}1.13 \\
(0.04)\end{array}$ & $\begin{array}{c}1.10 \\
(0.03)\end{array}$ & $\begin{array}{c}1.09 \\
(0.03)\end{array}$ & $\begin{array}{c}1.08 \\
(0.02)\end{array}$ & $\begin{array}{c}1.08 \\
(0.02)\end{array}$ & $\begin{array}{c}1.07 \\
(0.02)\end{array}$ & $\begin{array}{c}1.06 \\
(0.02)\end{array}$ & $\begin{array}{c}1.02 \\
(0.02)\end{array}$ & $\begin{array}{l}-0.17 \\
(0.05)\end{array}$ \\
\hline Size (SMB) & $\begin{array}{c}0.60 \\
(0.04)\end{array}$ & $\begin{array}{c}0.31 \\
(0.04)\end{array}$ & $\begin{array}{c}0.18 \\
(0.03)\end{array}$ & $\begin{array}{c}0.14 \\
(0.03)\end{array}$ & $\begin{array}{c}0.11 \\
(0.02)\end{array}$ & $\begin{array}{c}0.09 \\
(0.02)\end{array}$ & $\begin{array}{c}0.07 \\
(0.02)\end{array}$ & $\begin{array}{c}0.06 \\
(0.02)\end{array}$ & $\begin{array}{c}0.05 \\
(0.02)\end{array}$ & $\begin{array}{c}0.06 \\
(0.02)\end{array}$ & $\begin{array}{l}-0.55 \\
(0.04)\end{array}$ \\
\hline Value (HML) & $\begin{array}{c}0.39 \\
(0.20)\end{array}$ & $\begin{array}{c}0.15 \\
(0.18)\end{array}$ & $\begin{array}{c}0.13 \\
(0.14)\end{array}$ & $\begin{array}{c}0.07 \\
(0.13)\end{array}$ & $\begin{array}{c}0.04 \\
(0.12)\end{array}$ & $\begin{array}{c}0.03 \\
(0.12)\end{array}$ & $\begin{array}{c}0.03 \\
(0.11)\end{array}$ & $\begin{array}{c}0.01 \\
(0.11)\end{array}$ & $\begin{array}{c}0.00 \\
(0.10)\end{array}$ & $\begin{array}{l}-0.06 \\
(0.10)\end{array}$ & $\begin{array}{l}-0.45 \\
(0.15)\end{array}$ \\
\hline Momentum (MOM) & $\begin{array}{l}-0.39 \\
(0.07)\end{array}$ & $\begin{array}{l}-0.22 \\
(0.07)\end{array}$ & $\begin{array}{l}-0.21 \\
(0.05)\end{array}$ & $\begin{array}{l}-0.19 \\
(0.04)\end{array}$ & $\begin{array}{l}-0.18 \\
(0.04)\end{array}$ & $\begin{array}{l}-0.17 \\
(0.04)\end{array}$ & $\begin{array}{l}-0.15 \\
(0.03)\end{array}$ & $\begin{array}{l}-0.14 \\
(0.03)\end{array}$ & $\begin{array}{l}-0.12 \\
(0.03)\end{array}$ & $\begin{array}{l}-0.08 \\
(0.03)\end{array}$ & $\begin{array}{c}0.30 \\
(0.07)\end{array}$ \\
\hline Panel B: Risk and Returr & \multicolumn{10}{|c|}{ Account Value Deciles } & $\begin{array}{c}\text { Largest } \\
\text { minus }\end{array}$ \\
\hline & Smallest & 2 & 3 & 4 & 5 & 6 & 7 & 8 & & Largest & Smallest \\
\hline \multicolumn{12}{|l|}{ Realized: } \\
\hline Excess Returns & $\begin{array}{c}2.95 \% \\
(1.36 \%)\end{array}$ & $\begin{array}{c}2.03 \% \\
(1.13 \%)\end{array}$ & $\begin{array}{c}2.11 \% \\
(1.03 \%)\end{array}$ & $\begin{array}{c}1.95 \% \\
(0.97 \%)\end{array}$ & $\begin{array}{c}1.87 \% \\
(0.94 \%)\end{array}$ & $\begin{array}{c}1.81 \% \\
(0.93 \%)\end{array}$ & $\begin{array}{c}1.78 \% \\
(0.92 \%)\end{array}$ & $\begin{array}{c}1.75 \% \\
(0.90 \%)\end{array}$ & $\begin{array}{c}1.74 \% \\
(0.88 \%)\end{array}$ & $\begin{array}{c}1.66 \% \\
(0.84 \%)\end{array}$ & $\begin{array}{l}-1.29 \% \\
(0.79 \%)\end{array}$ \\
\hline Excess Log Returns & $\begin{array}{c}0.68 \% \\
(1.27 \%)\end{array}$ & $\begin{array}{c}0.63 \% \\
(1.12 \%)\end{array}$ & $\begin{array}{c}0.93 \% \\
(1.01 \%)\end{array}$ & $\begin{array}{c}0.95 \% \\
(0.96 \%)\end{array}$ & $\begin{array}{c}0.97 \% \\
(0.94 \%)\end{array}$ & $\begin{array}{c}0.99 \% \\
(0.93 \%)\end{array}$ & $\begin{array}{c}1.01 \% \\
(0.91 \%)\end{array}$ & $\begin{array}{c}1.02 \% \\
(0.90 \%)\end{array}$ & $\begin{array}{c}1.05 \% \\
(0.88 \%)\end{array}$ & $\begin{array}{c}0.99 \% \\
(0.84 \%)\end{array}$ & $\begin{array}{c}0.31 \% \\
(0.69 \%)\end{array}$ \\
\hline Excess Return Volatility & $\begin{array}{l}23.7 \% \\
(1.2 \%)\end{array}$ & $\begin{array}{l}17.6 \% \\
(0.9 \%)\end{array}$ & $\begin{array}{l}16.0 \% \\
(0.9 \%)\end{array}$ & $\begin{array}{l}14.5 \% \\
(0.9 \%)\end{array}$ & $\begin{array}{l}13.7 \% \\
(0.9 \%)\end{array}$ & $\begin{array}{l}13.0 \% \\
(0.9 \%)\end{array}$ & $\begin{array}{l}12.6 \% \\
(0.9 \%)\end{array}$ & $\begin{array}{l}12.2 \% \\
(0.9 \%)\end{array}$ & $\begin{array}{l}11.9 \% \\
(0.9 \%)\end{array}$ & $\begin{array}{l}11.6 \% \\
(0.8 \%)\end{array}$ & $\begin{array}{c}-12.1 \% \\
(1.0 \%)\end{array}$ \\
\hline Sharpe Ratio & $\begin{array}{c}0.12 \\
(0.05)\end{array}$ & $\begin{array}{c}0.12 \\
(0.06)\end{array}$ & $\begin{array}{c}0.13 \\
(0.06)\end{array}$ & $\begin{array}{c}0.13 \\
(0.07)\end{array}$ & $\begin{array}{c}0.14 \\
(0.07)\end{array}$ & $\begin{array}{c}0.14 \\
(0.07)\end{array}$ & $\begin{array}{c}0.14 \\
(0.07)\end{array}$ & $\begin{array}{c}0.14 \\
(0.07)\end{array}$ & $\begin{array}{c}0.15 \\
(0.07)\end{array}$ & $\begin{array}{c}0.14 \\
(0.07)\end{array}$ & $\begin{array}{c}0.02 \\
(0.04)\end{array}$ \\
\hline \multicolumn{12}{|c|}{ Long Run Global Factor Prices: } \\
\hline Excess Returns & $\begin{array}{c}0.48 \% \\
(0.73 \%)\end{array}$ & $\begin{array}{c}0.36 \% \\
(0.66 \%)\end{array}$ & $\begin{array}{c}0.62 \% \\
(0.58 \%)\end{array}$ & $\begin{array}{c}0.66 \% \\
(0.55 \%)\end{array}$ & $\begin{array}{c}0.68 \% \\
(0.52 \%)\end{array}$ & $\begin{array}{c}0.67 \% \\
(0.52 \%)\end{array}$ & $\begin{array}{c}0.67 \% \\
(0.50 \%)\end{array}$ & $\begin{array}{c}0.68 \% \\
(0.48 \%)\end{array}$ & $\begin{array}{c}0.71 \% \\
(0.46 \%)\end{array}$ & $\begin{array}{c}0.79 \% \\
(0.45 \%)\end{array}$ & $\begin{array}{c}0.31 \% \\
(0.43 \%)\end{array}$ \\
\hline Excess Log Returns & $\begin{array}{l}-1.82 \% \\
(0.74 \%)\end{array}$ & $\begin{array}{l}-1.05 \% \\
(0.68 \%)\end{array}$ & $\begin{array}{c}-0.56 \% \\
(0.59 \%)\end{array}$ & $\begin{array}{c}-0.35 \% \\
(0.56 \%)\end{array}$ & $\begin{array}{c}-0.22 \% \\
(0.54 \%)\end{array}$ & $\begin{array}{c}-0.15 \% \\
(0.53 \%)\end{array}$ & $\begin{array}{c}-0.11 \% \\
(0.51 \%)\end{array}$ & $\begin{array}{c}-0.06 \% \\
(0.49 \%)\end{array}$ & $\begin{array}{c}0.02 \% \\
(0.47 \%)\end{array}$ & $\begin{array}{c}0.12 \% \\
(0.46 \%)\end{array}$ & $\begin{array}{c}1.94 \% \\
(0.43 \%)\end{array}$ \\
\hline Sharpe Ratio & $\begin{array}{c}0.02 \\
(0.03)\end{array}$ & $\begin{array}{c}0.02 \\
(0.04)\end{array}$ & $\begin{array}{c}0.04 \\
(0.04)\end{array}$ & $\begin{array}{c}0.05 \\
(0.04)\end{array}$ & $\begin{array}{c}0.05 \\
(0.04)\end{array}$ & $\begin{array}{c}0.05 \\
(0.04)\end{array}$ & $\begin{array}{c}0.05 \\
(0.04)\end{array}$ & $\begin{array}{c}0.06 \\
(0.04)\end{array}$ & $\begin{array}{c}0.06 \\
(0.04)\end{array}$ & $\begin{array}{c}0.07 \\
(0.04)\end{array}$ & $\begin{array}{c}0.05 \\
(0.02)\end{array}$ \\
\hline \multicolumn{12}{|c|}{ Long Run Global Factor Prices, No Alpha: } \\
\hline Excess Returns & $\begin{array}{c}0.56 \% \\
(0.32 \%)\end{array}$ & $\begin{array}{c}0.55 \% \\
(0.29 \%)\end{array}$ & $\begin{array}{c}0.52 \% \\
(0.27 \%)\end{array}$ & $\begin{array}{c}0.49 \% \\
(0.27 \%)\end{array}$ & $\begin{array}{c}0.48 \% \\
(0.26 \%)\end{array}$ & $\begin{array}{c}0.48 \% \\
(0.26 \%)\end{array}$ & $\begin{array}{c}0.49 \% \\
(0.26 \%)\end{array}$ & $\begin{array}{c}0.49 \% \\
(0.26 \%)\end{array}$ & $\begin{array}{c}0.49 \% \\
(0.25 \%)\end{array}$ & $\begin{array}{c}0.47 \% \\
(0.24 \%)\end{array}$ & $\begin{array}{l}-0.09 \% \\
(0.14 \%)\end{array}$ \\
\hline Excess Log Returns & $\begin{array}{l}-1.74 \% \\
(0.37 \%)\end{array}$ & $\begin{array}{c}-0.86 \% \\
(0.32 \%)\end{array}$ & $\begin{array}{c}-0.67 \% \\
(0.30 \%)\end{array}$ & $\begin{array}{l}-0.52 \% \\
(0.29 \%)\end{array}$ & $\begin{array}{c}-0.42 \% \\
(0.28 \%)\end{array}$ & $\begin{array}{c}-0.35 \% \\
(0.28 \%)\end{array}$ & $\begin{array}{c}-0.29 \% \\
(0.28 \%)\end{array}$ & $\begin{array}{c}-0.25 \% \\
(0.27 \%)\end{array}$ & $\begin{array}{c}-0.21 \% \\
(0.27 \%)\end{array}$ & $\begin{array}{c}-0.20 \% \\
(0.26 \%)\end{array}$ & $\begin{array}{c}1.54 \% \\
(0.20 \%)\end{array}$ \\
\hline Sharpe & $\begin{array}{c}0.02 \\
(0.01)\end{array}$ & $\begin{array}{c}0.03 \\
(0.02)\end{array}$ & $\begin{array}{c}0.03 \\
(0.02)\end{array}$ & $\begin{array}{c}0.03 \\
(0.02)\end{array}$ & $\begin{array}{c}0.04 \\
(0.02)\end{array}$ & $\begin{array}{c}0.04 \\
(0.02)\end{array}$ & $\begin{array}{c}0.04 \\
(0.02)\end{array}$ & $\begin{array}{c}0.04 \\
(0.02)\end{array}$ & $\begin{array}{c}0.04 \\
(0.02)\end{array}$ & $\begin{array}{c}0.04 \\
(0.02)\end{array}$ & $\begin{array}{c}0.02 \\
(0.01)\end{array}$ \\
\hline
\end{tabular}


Table A5: Summary Statistics For Account Book Value: March 2002 - May 2011

Panel A presents means, minimums, maximums, and standard deviations of cross-sectional statistics spanning March 2002 through May 2011.

Panel B presents the time-series mean of cross-sectional correlations. Account growth, book value growth, log return, and inflow statistics are from the subset of accounts for which all three variables are available and book value is positive. Book value is defined as the account's cumulative net inflows, while alternative book value is the value of a hypothetical zero-return account with the same proportional distributions and amounts of new assets added.

Panel A: Time-series Variation in Cross-sectional Statistics

\begin{tabular}{lrrrr} 
& Mean & Minimum & Maximum & Standard Deviation \\
\hline Log Account Value & 10.80 & 10.32 & 11.53 & 0.26 \\
Log Book Value of Account & 10.68 & 10.32 & 10.93 & 0.18 \\
Log Alternative Book Value of Account & 10.69 & 10.39 & 10.94 & 0.15 \\
Fraction where Alternative Book $>0$ & $84.0 \%$ & $75.3 \%$ & $99.7 \%$ & $6.6 \%$ \\
StDev Log Account Value & 2.11 & 1.85 & 2.38 & 0.14 \\
StDev Log Book & 2.07 & 1.87 & 2.25 & 0.10 \\
StDev Log Alternative Book & 1.92 & 1.82 & 2.04 & 0.05 \\
\hline Log Account Returns & 0.0140 & -0.3711 & 0.3601 & 0.1016 \\
Account Growth & 0.0183 & -0.3252 & 0.3062 & 0.0833 \\
Book Growth & 0.0140 & -0.0426 & 0.1673 & 0.0328 \\
Alternative Book Growth & 0.0161 & -0.0541 & 0.1733 & 0.0347 \\
StDev Log Account Returns & 0.0920 & 0.0514 & 0.1636 & 0.0235 \\
StDev Account Growth & 0.4171 & 0.2810 & 0.6444 & 0.0804 \\
StDev Book Growth & 0.4091 & 0.2456 & 0.6549 & 0.0890 \\
StDev Alternative Book Growth & 0.3750 & 0.1730 & 0.6014 & 0.0980
\end{tabular}

Panel B: Average Cross-sectional Correlations

\begin{tabular}{|c|c|c|c|c|c|c|c|}
\hline Lagged Log Account Size Measures & $\begin{array}{c}\text { Account } \\
\text { Value }\end{array}$ & Book Value & $\begin{array}{l}\text { Alternative } \\
\text { Book Value }\end{array}$ & $\begin{array}{c}\text { Log } \\
\text { Account } \\
\text { Returns }\end{array}$ & $\begin{array}{l}\text { Account } \\
\text { Growth }\end{array}$ & $\begin{array}{c}\text { Book } \\
\text { Growth }\end{array}$ & $\begin{array}{c}\text { Alternative } \\
\text { Book } \\
\text { Growth } \\
\end{array}$ \\
\hline Account Value & 1.000 & 0.969 & 0.914 & 0.028 & -0.089 & -0.084 & -0.077 \\
\hline Book Value & & 1.000 & 0.951 & 0.020 & -0.090 & -0.089 & -0.080 \\
\hline Alternative Book Value & & & 1.000 & 0.017 & -0.088 & -0.089 & -0.102 \\
\hline \multicolumn{8}{|l|}{ Account Growth Measures } \\
\hline Log Account Returns & & & & 1.000 & 0.218 & -0.014 & -0.025 \\
\hline Account Growth & & & & & 1.000 & 0.931 & 0.754 \\
\hline Book Growth & & & & & & 1.000 & 0.807 \\
\hline Alternative Book Growth & & & & & & & 1.000 \\
\hline
\end{tabular}


Table A6: Risk and Return by Book Value of Account Deciles

Panel A replicates Table 2 using account-book-value sorted deciles under our definition of the book value of each account. Book value of account is equal to the value of each account under the counterfactual assumption that the account earns an Indian money market rate while having the same rupee inflows and the same proportional outflows that actually occurred. For accounts opened prior to the start of our data (February 2002), we assign an initial book value equal to the value of the account at the time. Panel B replicates Table 3 using book value of account deciles. Standard errors are reported in parentheses.

Panel A: Return Factor Loadings

Excess Return

gs

\begin{tabular}{|c|c|c|c|c|c|c|c|c|c|c|c|}
\hline \multirow[b]{2}{*}{ Excess Return } & llest & 2 & S & 4 & 3 & 0 & 7 & \multicolumn{3}{|r|}{9 Largest } & \multirow{2}{*}{$\begin{array}{c}\text { Smallest } \\
-1.29 \% \\
(0.69 \%)\end{array}$} \\
\hline & $\begin{array}{c}2.93 \% \\
(1.28 \%)\end{array}$ & $\begin{array}{c}2.09 \% \\
(1.12 \%)\end{array}$ & $\begin{array}{c}1.89 \% \\
(1.03 \%)\end{array}$ & $\begin{array}{c}1.90 \% \\
(0.97 \%)\end{array}$ & $\begin{array}{c}1.86 \% \\
(0.95 \%)\end{array}$ & $\begin{array}{c}1.80 \% \\
(0.93 \%)\end{array}$ & $\begin{array}{c}1.76 \% \\
(0.92 \%)\end{array}$ & $\begin{array}{c}1.74 \% \\
(0.90 \%)\end{array}$ & $\begin{array}{c}1.71 \% \\
(0.88 \%)\end{array}$ & $\begin{array}{c}1.65 \% \\
(0.83 \%)\end{array}$ & \\
\hline Four-Factor Alpha & $\begin{array}{c}-0.05 \% \\
(0.66 \%)\end{array}$ & $\begin{array}{l}-0.42 \% \\
(0.67 \%)\end{array}$ & $\begin{array}{l}-0.18 \% \\
(0.56 \%)\end{array}$ & $\begin{array}{c}0.14 \% \\
(0.50 \%)\end{array}$ & $\begin{array}{c}0.20 \% \\
(0.47 \%)\end{array}$ & $\begin{array}{c}0.18 \% \\
(0.45 \%)\end{array}$ & $\begin{array}{c}0.20 \% \\
(0.44 \%)\end{array}$ & $\begin{array}{c}0.22 \% \\
(0.41 \%)\end{array}$ & $\begin{array}{c}0.26 \% \\
(0.39 \%)\end{array}$ & $\begin{array}{c}0.37 \% \\
(0.37 \%)\end{array}$ & $\begin{array}{c}0.42 \% \\
(0.40 \%)\end{array}$ \\
\hline Market Beta & $\begin{array}{c}1.17 \\
(0.05)\end{array}$ & $\begin{array}{c}1.15 \\
(0.07)\end{array}$ & $\begin{array}{c}1.14 \\
(0.05)\end{array}$ & $\begin{array}{c}1.10 \\
(0.03)\end{array}$ & $\begin{array}{c}1.09 \\
(0.03)\end{array}$ & $\begin{array}{c}1.08 \\
(0.02)\end{array}$ & $\begin{array}{c}1.08 \\
(0.02)\end{array}$ & $\begin{array}{l}1.07 \\
(0.02)\end{array}$ & $\begin{array}{c}1.05 \\
(0.02)\end{array}$ & $\begin{array}{c}1.01 \\
(0.02)\end{array}$ & $\begin{array}{l}-0.16 \\
(0.04)\end{array}$ \\
\hline Size (SMB) & $\begin{array}{c}0.55 \\
(0.04)\end{array}$ & $\begin{array}{c}0.22 \\
(0.04)\end{array}$ & $\begin{array}{c}0.16 \\
(0.03)\end{array}$ & $\begin{array}{c}0.13 \\
(0.03)\end{array}$ & $\begin{array}{c}0.10 \\
(0.02)\end{array}$ & $\begin{array}{c}0.09 \\
(0.02)\end{array}$ & $\begin{array}{c}0.08 \\
(0.02)\end{array}$ & $\begin{array}{c}0.07 \\
(0.02)\end{array}$ & $\begin{array}{c}0.06 \\
(0.02)\end{array}$ & $\begin{array}{c}0.07 \\
(0.02)\end{array}$ & $\begin{array}{l}-0.48 \\
(0.04)\end{array}$ \\
\hline Value (HML) & $\begin{array}{c}0.39 \\
(0.18)\end{array}$ & $\begin{array}{c}0.28 \\
(0.17)\end{array}$ & $\begin{array}{c}0.15 \\
(0.15)\end{array}$ & $\begin{array}{c}0.06 \\
(0.14)\end{array}$ & $\begin{array}{c}0.04 \\
(0.13)\end{array}$ & $\begin{array}{c}0.03 \\
(0.12)\end{array}$ & $\begin{array}{c}0.01 \\
(0.12)\end{array}$ & $\begin{array}{c}0.00 \\
(0.11)\end{array}$ & $\begin{array}{l}-0.02 \\
(0.10)\end{array}$ & $\begin{array}{c}-0.08 \\
(0.10)\end{array}$ & $\begin{array}{l}-0.47 \\
(0.13)\end{array}$ \\
\hline Momentum (MOM) & $\begin{array}{l}-0.31 \\
(0.07)\end{array}$ & $\begin{array}{l}-0.14 \\
(0.08)\end{array}$ & $\begin{array}{l}-0.18 \\
(0.06)\end{array}$ & $\begin{array}{l}-0.19 \\
(0.04)\end{array}$ & $\begin{array}{l}-0.18 \\
(0.04)\end{array}$ & $\begin{array}{l}-0.17 \\
(0.04)\end{array}$ & $\begin{array}{l}-0.16 \\
(0.03)\end{array}$ & $\begin{array}{l}-0.15 \\
(0.03)\end{array}$ & $\begin{array}{l}-0.14 \\
(0.03)\end{array}$ & $\begin{array}{l}-0.11 \\
(0.03)\end{array}$ & $\begin{array}{c}0.20 \\
(0.06)\end{array}$ \\
\hline Panel B: Risk and Returns & \multicolumn{10}{|c|}{ Book Value of Account Deciles } & $\begin{array}{c}\text { Largest } \\
\text { minus }\end{array}$ \\
\hline & $\underline{\text { Smallest }}$ & 2 & 3 & 4 & 5 & 6 & 7 & 8 & & Largest & Smallest \\
\hline \multicolumn{12}{|l|}{ Realized: } \\
\hline Excess Returns & $\begin{array}{c}2.93 \% \\
(1.28 \%)\end{array}$ & $\begin{array}{c}2.09 \% \\
(1.12 \%)\end{array}$ & $\begin{array}{c}1.89 \% \\
(1.03 \%)\end{array}$ & $\begin{array}{c}1.90 \% \\
(0.97 \%)\end{array}$ & $\begin{array}{c}1.86 \% \\
(0.95 \%)\end{array}$ & $\begin{array}{c}1.80 \% \\
(0.93 \%)\end{array}$ & $\begin{array}{c}1.76 \% \\
(0.92 \%)\end{array}$ & $\begin{array}{c}1.74 \% \\
(0.90 \%)\end{array}$ & $\begin{array}{c}1.71 \% \\
(0.88 \%)\end{array}$ & $\begin{array}{c}1.65 \% \\
(0.83 \%)\end{array}$ & $\begin{array}{l}-1.29 \% \\
(0.69 \%)\end{array}$ \\
\hline Excess Log Returns & $\begin{array}{c}0.79 \% \\
(1.21 \%)\end{array}$ & $\begin{array}{c}0.71 \% \\
(1.12 \%)\end{array}$ & $\begin{array}{c}0.73 \% \\
(1.03 \%)\end{array}$ & $\begin{array}{c}0.91 \% \\
(0.96 \%)\end{array}$ & $\begin{array}{c}0.96 \% \\
(0.94 \%)\end{array}$ & $\begin{array}{c}0.98 \% \\
(0.92 \%)\end{array}$ & $\begin{array}{c}0.99 \% \\
(0.91 \%)\end{array}$ & $\begin{array}{c}1.02 \% \\
(0.90 \%)\end{array}$ & $\begin{array}{c}1.04 \% \\
(0.87 \%)\end{array}$ & $\begin{array}{c}1.02 \% \\
(0.83 \%)\end{array}$ & $\begin{array}{c}0.23 \% \\
(0.61 \%)\end{array}$ \\
\hline Excess Return Volatility & $\begin{array}{l}22.9 \% \\
(1.1 \%)\end{array}$ & $\begin{array}{l}17.5 \% \\
(1.0 \%)\end{array}$ & $\begin{array}{l}15.7 \% \\
(0.9 \%)\end{array}$ & $\begin{array}{l}14.5 \% \\
(0.9 \%)\end{array}$ & $\begin{array}{l}13.7 \% \\
(0.9 \%)\end{array}$ & $\begin{array}{l}13.1 \% \\
(0.9 \%)\end{array}$ & $\begin{array}{l}12.6 \% \\
(0.9 \%)\end{array}$ & $\begin{array}{l}12.2 \% \\
(0.9 \%)\end{array}$ & $\begin{array}{l}11.7 \% \\
(0.9 \%)\end{array}$ & $\begin{array}{l}11.3 \% \\
(0.8 \%)\end{array}$ & $\begin{array}{c}-11.6 \% \\
(0.9 \%)\end{array}$ \\
\hline Sharpe Ratio & $\begin{array}{c}0.13 \\
(0.05)\end{array}$ & $\begin{array}{c}0.12 \\
(0.06)\end{array}$ & $\begin{array}{c}0.12 \\
(0.06)\end{array}$ & $\begin{array}{c}0.13 \\
(0.07)\end{array}$ & $\begin{array}{c}0.14 \\
(0.07)\end{array}$ & $\begin{array}{c}0.14 \\
(0.07)\end{array}$ & $\begin{array}{c}0.14 \\
(0.07)\end{array}$ & $\begin{array}{c}0.14 \\
(0.07)\end{array}$ & $\begin{array}{c}0.15 \\
(0.07)\end{array}$ & $\begin{array}{c}0.15 \\
(0.07)\end{array}$ & $\begin{array}{c}0.02 \\
(0.04)\end{array}$ \\
\hline \multicolumn{12}{|c|}{ Long Run Global Factor Prices: } \\
\hline Excess Returns & $\begin{array}{c}0.54 \% \\
(0.70 \%)\end{array}$ & $\begin{array}{c}0.21 \% \\
(0.69 \%)\end{array}$ & $\begin{array}{c}0.37 \% \\
(0.60 \%)\end{array}$ & $\begin{array}{c}0.63 \% \\
(0.56 \%)\end{array}$ & $\begin{array}{c}0.68 \% \\
(0.53 \%)\end{array}$ & $\begin{array}{c}0.66 \% \\
(0.51 \%)\end{array}$ & $\begin{array}{c}0.67 \% \\
(0.50 \%)\end{array}$ & $\begin{array}{c}0.69 \% \\
(0.48 \%)\end{array}$ & $\begin{array}{c}0.73 \% \\
(0.46 \%)\end{array}$ & $\begin{array}{c}0.82 \% \\
(0.44 \%)\end{array}$ & $\begin{array}{c}0.28 \% \\
(0.40 \%)\end{array}$ \\
\hline Excess Log Returns & $\begin{array}{l}-1.63 \% \\
(0.72 \%)\end{array}$ & $\begin{array}{l}-1.19 \% \\
(0.72 \%)\end{array}$ & $\begin{array}{l}-0.80 \% \\
(0.62 \%)\end{array}$ & $\begin{array}{c}-0.37 \% \\
(0.57 \%)\end{array}$ & $\begin{array}{l}-0.23 \% \\
(0.54 \%)\end{array}$ & $\begin{array}{c}-0.17 \% \\
(0.53 \%)\end{array}$ & $\begin{array}{c}-0.10 \% \\
(0.52 \%)\end{array}$ & $\begin{array}{c}-0.03 \% \\
(0.49 \%)\end{array}$ & $\begin{array}{c}0.05 \% \\
(0.47 \%)\end{array}$ & $\begin{array}{c}0.19 \% \\
(0.45 \%)\end{array}$ & $\begin{array}{c}1.82 \% \\
(0.40 \%)\end{array}$ \\
\hline Sharpe Ratio & $\begin{array}{c}0.02 \\
(0.03)\end{array}$ & $\begin{array}{c}0.01 \\
(0.04)\end{array}$ & $\begin{array}{c}0.02 \\
(0.04)\end{array}$ & $\begin{array}{c}0.04 \\
(0.04)\end{array}$ & $\begin{array}{c}0.05 \\
(0.04)\end{array}$ & $\begin{array}{c}0.05 \\
(0.04)\end{array}$ & $\begin{array}{c}0.05 \\
(0.04)\end{array}$ & $\begin{array}{c}0.06 \\
(0.04)\end{array}$ & $\begin{array}{c}0.06 \\
(0.04)\end{array}$ & $\begin{array}{c}0.07 \\
(0.04)\end{array}$ & $\begin{array}{c}0.05 \\
(0.02)\end{array}$ \\
\hline \multicolumn{12}{|c|}{ Long Run Global Factor Prices, No Alpha: } \\
\hline Excess Returns & $\begin{array}{c}0.59 \% \\
(0.30 \%)\end{array}$ & $\begin{array}{c}0.63 \% \\
(0.28 \%)\end{array}$ & $\begin{array}{c}0.55 \% \\
(0.27 \%)\end{array}$ & $\begin{array}{c}0.49 \% \\
(0.27 \%)\end{array}$ & $\begin{array}{c}0.48 \% \\
(0.26 \%)\end{array}$ & $\begin{array}{c}0.48 \% \\
(0.26 \%)\end{array}$ & $\begin{array}{c}0.48 \% \\
(0.26 \%)\end{array}$ & $\begin{array}{c}0.47 \% \\
(0.26 \%)\end{array}$ & $\begin{array}{c}0.47 \% \\
(0.25 \%)\end{array}$ & $\begin{array}{c}0.44 \% \\
(0.24 \%)\end{array}$ & $\begin{array}{l}-0.14 \% \\
(0.12 \%)\end{array}$ \\
\hline Excess Log Returns & $\begin{array}{l}-1.58 \% \\
(0.35 \%)\end{array}$ & $\begin{array}{l}-0.77 \% \\
(0.32 \%)\end{array}$ & $\begin{array}{c}-0.62 \% \\
(0.31 \%)\end{array}$ & $\begin{array}{l}-0.51 \% \\
(0.29 \%)\end{array}$ & $\begin{array}{c}-0.42 \% \\
(0.29 \%)\end{array}$ & $\begin{array}{c}-0.36 \% \\
(0.28 \%)\end{array}$ & $\begin{array}{c}-0.30 \% \\
(0.28 \%)\end{array}$ & $\begin{array}{l}-0.25 \% \\
(0.28 \%)\end{array}$ & $\begin{array}{l}-0.21 \% \\
(0.27 \%)\end{array}$ & $\begin{array}{l}-0.19 \% \\
(0.26 \%)\end{array}$ & $\begin{array}{c}1.39 \% \\
(0.16 \%)\end{array}$ \\
\hline Sharpe Ratio & $\begin{array}{c}0.03 \\
(0.01)\end{array}$ & $\begin{array}{c}0.04 \\
(0.02)\end{array}$ & $\begin{array}{c}0.03 \\
(0.02)\end{array}$ & $\begin{array}{c}0.03 \\
(0.02)\end{array}$ & $\begin{array}{c}0.04 \\
(0.02)\end{array}$ & $\begin{array}{c}0.04 \\
(0.02)\end{array}$ & $\begin{array}{c}0.04 \\
(0.02)\end{array}$ & $\begin{array}{c}0.04 \\
(0.02)\end{array}$ & $\begin{array}{c}0.04 \\
(0.02)\end{array}$ & $\begin{array}{c}0.04 \\
(0.02)\end{array}$ & $\begin{array}{c}0.01 \\
(0.01)\end{array}$ \\
\hline
\end{tabular}


Table A7: Risk and Return by Alternative Book Value of Account Deciles

Table A7 replicates Table A6 using an alternative definition of the book value of an account. Under this alternative definition, the book value of an account is defined as the cumulative value of purchases minus sales of stock in the account. For accounts opened prior to the start of our data (February 2002), we assign an initial book value equal to the value of the account at the time. Standard errors are reported in parentheses.

\begin{tabular}{|c|c|c|c|c|c|c|c|c|c|c|c|}
\hline \multicolumn{11}{|c|}{ Panel A: Return Factor Loadings } & \multirow{2}{*}{$\begin{array}{c}\text { Largest } \\
\text { minus } \\
\text { Smallest }\end{array}$} \\
\hline & Smallest & 2 & 3 & 4 & 5 & 6 & 7 & 8 & \multicolumn{2}{|r|}{9 Largest } & \\
\hline Excess Return & $\begin{array}{c}2.44 \% \\
(1.11 \%)\end{array}$ & $\begin{array}{c}2.51 \% \\
(1.11 \%)\end{array}$ & $\begin{array}{c}2.21 \% \\
(1.06 \%)\end{array}$ & $\begin{array}{c}1.73 \% \\
(1.04 \%)\end{array}$ & $\begin{array}{c}1.83 \% \\
(0.95 \%)\end{array}$ & $\begin{array}{c}1.80 \% \\
(0.94 \%)\end{array}$ & $\begin{array}{c}1.75 \% \\
(0.92 \%)\end{array}$ & $\begin{array}{c}1.71 \% \\
(0.91 \%)\end{array}$ & $\begin{array}{c}1.70 \% \\
(0.88 \%)\end{array}$ & $\begin{array}{c}1.63 \% \\
\quad(0.83 \%)\end{array}$ & $\begin{array}{l}-0.81 \% \\
(0.48 \%)\end{array}$ \\
\hline Four-Factor Alpha & $\begin{array}{l}-0.06 \% \\
(0.64 \%)\end{array}$ & $\begin{array}{c}0.12 \% \\
(0.56 \%)\end{array}$ & $\begin{array}{c}-0.09 \% \\
(0.56 \%)\end{array}$ & $\begin{array}{c}-0.26 \% \\
(0.58 \%)\end{array}$ & $\begin{array}{c}0.13 \% \\
(0.48 \%)\end{array}$ & $\begin{array}{c}0.18 \% \\
(0.46 \%)\end{array}$ & $\begin{array}{c}0.18 \% \\
(0.44 \%)\end{array}$ & $\begin{array}{c}0.19 \% \\
(0.41 \%)\end{array}$ & $\begin{array}{c}0.26 \% \\
(0.39 \%)\end{array}$ & $\begin{array}{c}0.36 \% \\
(0.37 \%)\end{array}$ & $\begin{array}{c}0.42 \% \\
(0.35 \%)\end{array}$ \\
\hline Market Beta & $\begin{array}{c}1.11 \\
(0.04)\end{array}$ & $\begin{array}{c}1.14 \\
(0.04)\end{array}$ & $\begin{array}{c}1.13 \\
(0.04)\end{array}$ & $\begin{array}{c}1.14 \\
(0.06)\end{array}$ & $\begin{array}{c}1.09 \\
(0.03)\end{array}$ & $\begin{array}{c}1.09 \\
(0.03)\end{array}$ & $\begin{array}{c}1.08 \\
(0.02)\end{array}$ & $\begin{array}{c}1.07 \\
(0.02)\end{array}$ & $\begin{array}{c}1.06 \\
(0.02)\end{array}$ & $\begin{array}{c}1.01 \\
(0.02)\end{array}$ & $\begin{array}{l}-0.09 \\
(0.04)\end{array}$ \\
\hline Size (SMB) & $\begin{array}{c}0.36 \\
(0.03)\end{array}$ & $\begin{array}{c}0.36 \\
(0.03)\end{array}$ & $\begin{array}{c}0.23 \\
(0.04)\end{array}$ & $\begin{array}{c}0.12 \\
(0.03)\end{array}$ & $\begin{array}{c}0.10 \\
(0.02)\end{array}$ & $\begin{array}{c}0.08 \\
(0.02)\end{array}$ & $\begin{array}{c}0.07 \\
(0.02)\end{array}$ & $\begin{array}{c}0.06 \\
(0.02)\end{array}$ & $\begin{array}{c}0.05 \\
(0.02)\end{array}$ & $\begin{array}{c}0.06 \\
(0.02)\end{array}$ & $\begin{array}{l}-0.30 \\
(0.03)\end{array}$ \\
\hline Value (HML) & $\begin{array}{c}0.28 \\
(0.18)\end{array}$ & $\begin{array}{c}0.22 \\
(0.15)\end{array}$ & $\begin{array}{c}0.22 \\
(0.15)\end{array}$ & $\begin{array}{c}0.12 \\
(0.15)\end{array}$ & $\begin{array}{c}0.05 \\
(0.13)\end{array}$ & $\begin{array}{c}0.03 \\
(0.12)\end{array}$ & $\begin{array}{c}0.02 \\
(0.12)\end{array}$ & $\begin{array}{c}0.00 \\
(0.11)\end{array}$ & $\begin{array}{l}-0.02 \\
(0.10)\end{array}$ & $\begin{array}{l}-0.07 \\
(0.10)\end{array}$ & $\begin{array}{l}-0.35 \\
(0.12)\end{array}$ \\
\hline Momentum (MOM) & $\begin{array}{l}-0.23 \\
(0.06)\end{array}$ & $\begin{array}{l}-0.21 \\
(0.06)\end{array}$ & $\begin{array}{l}-0.18 \\
(0.06)\end{array}$ & $\begin{array}{l}-0.14 \\
(0.07)\end{array}$ & $\begin{array}{l}-0.18 \\
(0.04)\end{array}$ & $\begin{array}{l}-0.19 \\
(0.04)\end{array}$ & $\begin{array}{l}-0.18 \\
(0.04)\end{array}$ & $\begin{array}{l}-0.17 \\
(0.03)\end{array}$ & $\begin{array}{l}-0.15 \\
(0.03)\end{array}$ & $\begin{array}{l}-0.12 \\
(0.03)\end{array}$ & $\begin{array}{c}0.11 \\
(0.06)\end{array}$ \\
\hline Panel B: Risk and Returns & \multicolumn{10}{|c|}{ Book Value of Account Deciles } & $\begin{array}{c}\text { Largest } \\
\text { minus }\end{array}$ \\
\hline & Smallest & 2 & 3 & 4 & 5 & 6 & 7 & 8 & & 9 Largest & Smallest \\
\hline \multicolumn{12}{|l|}{ Realized: } \\
\hline Excess Returns & $\begin{array}{c}2.44 \% \\
(1.11 \%)\end{array}$ & $\begin{array}{c}2.51 \% \\
(1.11 \%)\end{array}$ & $\begin{array}{c}2.21 \% \\
(1.06 \%)\end{array}$ & $\begin{array}{c}1.73 \% \\
(1.04 \%)\end{array}$ & $\begin{array}{c}1.83 \% \\
(0.95 \%)\end{array}$ & $\begin{array}{c}1.80 \% \\
(0.94 \%)\end{array}$ & $\begin{array}{c}1.75 \% \\
(0.92 \%)\end{array}$ & $\begin{array}{c}1.71 \% \\
(0.91 \%)\end{array}$ & $\begin{array}{c}1.70 \% \\
(0.88 \%)\end{array}$ & $\begin{array}{c}1.63 \% \\
(0.83 \%)\end{array}$ & $\begin{array}{l}-0.81 \% \\
(0.48 \%)\end{array}$ \\
\hline Excess Log Returns & $\begin{array}{c}0.99 \% \\
(1.06 \%)\end{array}$ & $\begin{array}{c}1.00 \% \\
(1.06 \%)\end{array}$ & $\begin{array}{c}0.86 \% \\
(1.04 \%)\end{array}$ & $\begin{array}{c}0.63 \% \\
(1.05 \%)\end{array}$ & $\begin{array}{c}0.88 \% \\
(0.95 \%)\end{array}$ & $\begin{array}{c}0.94 \% \\
(0.93 \%)\end{array}$ & $\begin{array}{c}0.96 \% \\
(0.92 \%)\end{array}$ & $\begin{array}{c}0.97 \% \\
(0.90 \%)\end{array}$ & $\begin{array}{c}1.01 \% \\
(0.88 \%)\end{array}$ & $\begin{array}{l}1.00 \% \\
(0.83 \%)\end{array}$ & $\begin{array}{c}0.01 \% \\
(0.43 \%)\end{array}$ \\
\hline Excess Return Volatility & $\begin{array}{l}18.3 \% \\
(1.0 \%)\end{array}$ & $\begin{array}{l}18.7 \% \\
(1.0 \%)\end{array}$ & $\begin{array}{l}17.4 \% \\
(0.9 \%)\end{array}$ & $\begin{array}{l}15.3 \% \\
(0.9 \%)\end{array}$ & $\begin{array}{l}14.2 \% \\
(0.9 \%)\end{array}$ & $\begin{array}{l}13.4 \% \\
(0.9 \%)\end{array}$ & $\begin{array}{l}12.8 \% \\
(0.9 \%)\end{array}$ & $\begin{array}{l}12.4 \% \\
(0.9 \%)\end{array}$ & $\begin{array}{l}11.9 \% \\
(0.9 \%)\end{array}$ & $\begin{array}{l}11.4 \% \\
(0.8 \%)\end{array}$ & $\begin{array}{l}-7.0 \% \\
(0.8 \%)\end{array}$ \\
\hline Sharpe Ratio & $\begin{array}{c}0.13 \\
(0.06)\end{array}$ & $\begin{array}{c}0.13 \\
(0.06)\end{array}$ & $\begin{array}{c}0.13 \\
(0.06)\end{array}$ & $\begin{array}{c}0.11 \\
(0.07)\end{array}$ & $\begin{array}{c}0.13 \\
(0.07)\end{array}$ & $\begin{array}{c}0.13 \\
(0.07)\end{array}$ & $\begin{array}{c}0.14 \\
(0.07)\end{array}$ & $\begin{array}{c}0.14 \\
(0.07)\end{array}$ & $\begin{array}{c}0.14 \\
(0.07)\end{array}$ & $\begin{array}{c}0.14 \\
(0.07)\end{array}$ & $\begin{array}{c}0.01 \\
(0.03)\end{array}$ \\
\hline \multicolumn{12}{|c|}{ Long Run Global Factor Prices: } \\
\hline xcess Returns & $\begin{array}{c}0.50 \% \\
(0.68 \%)\end{array}$ & $\begin{array}{c}0.68 \% \\
(0.61 \%)\end{array}$ & $\begin{array}{c}0.49 \% \\
(0.60 \%)\end{array}$ & $\begin{array}{c}0.30 \% \\
(0.62 \%)\end{array}$ & $\begin{array}{c}0.62 \% \\
(0.54 \%)\end{array}$ & $\begin{array}{c}0.66 \% \\
(0.52 \%)\end{array}$ & $\begin{array}{c}0.65 \% \\
(0.51 \%)\end{array}$ & $\begin{array}{c}0.66 \% \\
(0.48 \%)\end{array}$ & $\begin{array}{c}0.72 \% \\
(0.46 \%)\end{array}$ & $\begin{array}{c}0.80 \% \\
(0.44 \%)\end{array}$ & $\begin{array}{c}0.30 \% \\
(0.34 \%)\end{array}$ \\
\hline Excess Log Returns & $\begin{array}{c}-0.97 \% \\
(0.69 \%)\end{array}$ & $\begin{array}{c}-0.85 \% \\
(0.62 \%)\end{array}$ & $\begin{array}{c}-0.88 \% \\
(0.62 \%)\end{array}$ & $\begin{array}{c}-0.81 \% \\
(0.65 \%)\end{array}$ & $\begin{array}{c}-0.34 \% \\
(0.55 \%)\end{array}$ & $\begin{array}{c}-0.21 \% \\
(0.53 \%)\end{array}$ & $\begin{array}{c}-0.15 \% \\
(0.52 \%)\end{array}$ & $\begin{array}{c}-0.09 \% \\
(0.49 \%)\end{array}$ & $\begin{array}{c}0.03 \% \\
(0.48 \%)\end{array}$ & $\begin{array}{c}0.16 \% \\
(0.45 \%)\end{array}$ & $\begin{array}{c}1.13 \% \\
(0.35 \%)\end{array}$ \\
\hline Sharpe Ratio & $\begin{array}{c}0.03 \\
(0.04)\end{array}$ & $\begin{array}{c}0.04 \\
(0.03)\end{array}$ & $\begin{array}{c}0.03 \\
(0.03)\end{array}$ & $\begin{array}{c}0.02 \\
(0.04)\end{array}$ & $\begin{array}{c}0.04 \\
(0.04)\end{array}$ & $\begin{array}{c}0.05 \\
(0.04)\end{array}$ & $\begin{array}{c}0.05 \\
(0.04)\end{array}$ & $\begin{array}{c}0.05 \\
(0.04)\end{array}$ & $\begin{array}{c}0.06 \\
(0.04)\end{array}$ & $\begin{array}{c}0.07 \\
(0.04)\end{array}$ & $\begin{array}{c}0.04 \\
(0.02)\end{array}$ \\
\hline \multicolumn{12}{|c|}{ Long Run Global Factor Prices, No Alpha: } \\
\hline Excess Returns & $\begin{array}{c}0.56 \% \\
(0.28 \%)\end{array}$ & $\begin{array}{c}0.56 \% \\
(0.28 \%)\end{array}$ & $\begin{array}{c}0.58 \% \\
(0.27 \%)\end{array}$ & $\begin{array}{c}0.56 \% \\
(0.27 \%)\end{array}$ & $\begin{array}{c}0.49 \% \\
(0.26 \%)\end{array}$ & $\begin{array}{c}0.47 \% \\
(0.26 \%)\end{array}$ & $\begin{array}{c}0.47 \% \\
(0.26 \%)\end{array}$ & $\begin{array}{c}0.47 \% \\
(0.26 \%)\end{array}$ & $\begin{array}{c}0.46 \% \\
(0.25 \%)\end{array}$ & $\begin{array}{c}0.44 \% \\
(0.24 \%)\end{array}$ & $\begin{array}{c}-0.12 \% \\
(0.09 \%)\end{array}$ \\
\hline Excess Log Returns & $\begin{array}{c}-0.91 \% \\
(0.31 \%)\end{array}$ & $\begin{array}{c}-0.97 \% \\
(0.31 \%)\end{array}$ & $\begin{array}{l}-0.80 \% \\
(0.31 \%)\end{array}$ & $\begin{array}{c}-0.54 \% \\
(0.31 \%)\end{array}$ & $\begin{array}{c}-0.47 \% \\
(0.29 \%)\end{array}$ & $\begin{array}{c}-0.40 \% \\
(0.28 \%)\end{array}$ & $\begin{array}{c}-0.33 \% \\
(0.28 \%)\end{array}$ & $\begin{array}{c}-0.28 \% \\
(0.28 \%)\end{array}$ & $\begin{array}{l}-0.23 \% \\
(0.27 \%)\end{array}$ & $\begin{array}{c}-0.20 \% \\
(0.26 \%)\end{array}$ & $\begin{array}{c}0.71 \% \\
(0.11 \%)\end{array}$ \\
\hline Sharpe Ratio & $\begin{array}{c}0.03 \\
(0.02)\end{array}$ & $\begin{array}{c}0.03 \\
(0.02)\end{array}$ & $\begin{array}{c}0.03 \\
(0.02)\end{array}$ & $\begin{array}{c}0.04 \\
(0.02)\end{array}$ & $\begin{array}{c}0.03 \\
(0.02)\end{array}$ & $\begin{array}{c}0.04 \\
(0.02)\end{array}$ & $\begin{array}{c}0.04 \\
(0.02)\end{array}$ & $\begin{array}{c}0.04 \\
(0.02)\end{array}$ & $\begin{array}{c}0.04 \\
(0.02)\end{array}$ & $\begin{array}{c}0.04 \\
(0.02)\end{array}$ & $\begin{array}{c}0.01 \\
(0.01)\end{array}$ \\
\hline
\end{tabular}


Table A8: Risk and Return by Account Value Deciles, Excluding Returns on Micro-Cap Stocks

Panel A replicates Table 2 under the exclusion of returns on stocks whose aggregate holdings in NSDL at the end of the prior month are less than 500 million Rs (roughly \$10 million). Panel B replicates Table 3 also excluding these micro-cap stock returns. Standard errors are reported in parentheses.

\begin{tabular}{|c|c|c|c|c|c|c|c|c|c|c|c|}
\hline \multicolumn{11}{|c|}{ Panel A: Return Factor Loadings } & \multirow{2}{*}{$\begin{array}{c}\text { Largest } \\
\text { minus } \\
\text { Smallest }\end{array}$} \\
\hline \multirow[b]{2}{*}{ Excess Return } & Smallest & 2 & 3 & 4 & 5 & 6 & 7 & 8 & & 9 Largest & \\
\hline & $\begin{array}{c}2.19 \% \\
(1.23 \%)\end{array}$ & $\begin{array}{c}1.70 \% \\
(1.10 \%)\end{array}$ & $\begin{array}{c}2.00 \% \\
(1.01 \%)\end{array}$ & $\begin{array}{c}1.85 \% \\
(0.96 \%)\end{array}$ & $\begin{array}{c}1.78 \% \\
(0.94 \%)\end{array}$ & $\begin{array}{c}1.78 \% \\
(0.92 \%)\end{array}$ & $\begin{array}{c}1.74 \% \\
(0.90 \%)\end{array}$ & $\begin{array}{c}1.69 \% \\
(0.88 \%)\end{array}$ & $\begin{array}{c}1.71 \% \\
(0.86 \%)\end{array}$ & $\begin{array}{c}1.66 \% \\
(0.81 \%)\end{array}$ & $\begin{array}{l}-0.53 \% \\
(0.64 \%)\end{array}$ \\
\hline Four-Factor Alpha & $\begin{array}{c}-0.10 \% \\
(0.71 \%)\end{array}$ & $\begin{array}{c}-0.30 \% \\
(0.69 \%)\end{array}$ & $\begin{array}{c}0.09 \% \\
(0.54 \%)\end{array}$ & $\begin{array}{c}0.19 \% \\
(0.50 \%)\end{array}$ & $\begin{array}{c}0.24 \% \\
(0.47 \%)\end{array}$ & $\begin{array}{c}0.27 \% \\
(0.45 \%)\end{array}$ & $\begin{array}{c}0.25 \% \\
(0.43 \%)\end{array}$ & $\begin{array}{c}0.26 \% \\
(0.40 \%)\end{array}$ & $\begin{array}{c}0.33 \% \\
(0.38 \%)\end{array}$ & $\begin{array}{c}0.43 \% \\
(0.36 \%)\end{array}$ & $\begin{array}{c}0.54 \% \\
(0.46 \%)\end{array}$ \\
\hline Market Beta & $\begin{array}{c}1.21 \\
(0.06)\end{array}$ & $\begin{array}{c}1.16 \\
(0.08)\end{array}$ & $\begin{array}{c}1.14 \\
(0.04)\end{array}$ & $\begin{array}{c}1.10 \\
(0.03)\end{array}$ & $\begin{array}{c}1.10 \\
(0.03)\end{array}$ & $\begin{array}{c}1.08 \\
(0.02)\end{array}$ & $\begin{array}{c}1.08 \\
(0.02)\end{array}$ & $\begin{array}{c}1.06 \\
(0.02)\end{array}$ & $\begin{array}{c}1.05 \\
(0.02)\end{array}$ & $\begin{array}{c}1.01 \\
(0.02)\end{array}$ & $\begin{array}{l}-0.21 \\
(0.05)\end{array}$ \\
\hline Size (SMB) & $\begin{array}{c}0.31 \\
(0.05)\end{array}$ & $\begin{array}{c}0.16 \\
(0.05)\end{array}$ & $\begin{array}{c}0.07 \\
(0.03)\end{array}$ & $\begin{array}{c}0.06 \\
(0.03)\end{array}$ & $\begin{array}{c}0.05 \\
(0.02)\end{array}$ & $\begin{array}{c}0.04 \\
(0.02)\end{array}$ & $\begin{array}{c}0.03 \\
(0.02)\end{array}$ & $\begin{array}{c}0.03 \\
(0.02)\end{array}$ & $\begin{array}{c}0.03 \\
(0.02)\end{array}$ & $\begin{array}{c}0.03 \\
(0.02)\end{array}$ & $\begin{array}{l}-0.28 \\
(0.05)\end{array}$ \\
\hline Value (HML) & $\begin{array}{c}0.18 \\
(0.21)\end{array}$ & $\begin{array}{c}0.10 \\
(0.20)\end{array}$ & $\begin{array}{c}0.12 \\
(0.14)\end{array}$ & $\begin{array}{c}0.05 \\
(0.14)\end{array}$ & $\begin{array}{c}0.00 \\
(0.13)\end{array}$ & $\begin{array}{c}0.00 \\
(0.12)\end{array}$ & $\begin{array}{l}-0.01 \\
(0.11)\end{array}$ & $\begin{array}{l}-0.02 \\
(0.11)\end{array}$ & $\begin{array}{l}-0.04 \\
(0.10)\end{array}$ & $\begin{array}{l}-0.09 \\
(0.09)\end{array}$ & $\begin{array}{l}-0.28 \\
(0.16)\end{array}$ \\
\hline Momentum (MOM) & $\begin{array}{l}-0.35 \\
(0.09)\end{array}$ & $\begin{array}{l}-0.17 \\
(0.09)\end{array}$ & $\begin{array}{l}-0.19 \\
(0.06)\end{array}$ & $\begin{array}{l}-0.18 \\
(0.05)\end{array}$ & $\begin{array}{l}-0.17 \\
(0.04)\end{array}$ & $\begin{array}{l}-0.16 \\
(0.04)\end{array}$ & $\begin{array}{l}-0.14 \\
(0.03)\end{array}$ & $\begin{array}{c}-0.13 \\
(0.03)\end{array}$ & $\begin{array}{c}-0.11 \\
(0.03)\end{array}$ & $\begin{array}{l}-0.07 \\
(0.03)\end{array}$ & $\begin{array}{c}0.28 \\
(0.08)\end{array}$ \\
\hline \multirow[t]{2}{*}{ Panel B: Risk and Returns } & \multicolumn{10}{|c|}{ Account Value Deciles } & $\begin{array}{c}\text { Largest } \\
\text { minus }\end{array}$ \\
\hline & Smallest & 2 & 3 & 4 & 5 & 6 & 7 & 0 & & 9 Largest & Smallest \\
\hline \multicolumn{12}{|l|}{ Realized: } \\
\hline Excess Returns & $\begin{array}{c}2.19 \% \\
(1.23 \%)\end{array}$ & $\begin{array}{c}1.70 \% \\
(1.10 \%)\end{array}$ & $\begin{array}{c}2.00 \% \\
(1.01 \%)\end{array}$ & $\begin{array}{c}1.85 \% \\
(0.96 \%)\end{array}$ & $\begin{array}{c}1.78 \% \\
(0.94 \%)\end{array}$ & $\begin{array}{c}1.78 \% \\
(0.92 \%)\end{array}$ & $\begin{array}{c}1.74 \% \\
(0.90 \%)\end{array}$ & $\begin{array}{c}1.69 \% \\
(0.88 \%)\end{array}$ & $\begin{array}{c}1.71 \% \\
(0.86 \%)\end{array}$ & $\begin{array}{c}1.66 \% \\
(0.81 \%)\end{array}$ & $\begin{array}{l}-0.53 \% \\
(0.64 \%)\end{array}$ \\
\hline Excess Log Returns & $\begin{array}{c}0.47 \% \\
(1.18 \%)\end{array}$ & $\begin{array}{c}0.48 \% \\
(1.11 \%)\end{array}$ & $\begin{array}{c}0.90 \% \\
(1.00 \%)\end{array}$ & $\begin{array}{c}0.90 \% \\
(0.95 \%)\end{array}$ & $\begin{array}{c}0.92 \% \\
(0.93 \%)\end{array}$ & $\begin{array}{c}0.98 \% \\
(0.92 \%)\end{array}$ & $\begin{array}{c}1.00 \% \\
(0.90 \%)\end{array}$ & $\begin{array}{c}1.01 \% \\
(0.88 \%)\end{array}$ & $\begin{array}{c}1.07 \% \\
(0.86 \%)\end{array}$ & $\begin{array}{c}1.08 \% \\
(0.81 \%)\end{array}$ & $\begin{array}{c}0.62 \% \\
(0.58 \%)\end{array}$ \\
\hline Excess Return Volatility & $\begin{array}{l}19.8 \% \\
(1.1 \%)\end{array}$ & $\begin{array}{l}16.0 \% \\
(1.0 \%)\end{array}$ & $\begin{array}{l}15.3 \% \\
(0.9 \%)\end{array}$ & $\begin{array}{l}14.1 \% \\
(0.9 \%)\end{array}$ & $\begin{array}{l}13.3 \% \\
(0.9 \%)\end{array}$ & $\begin{array}{l}12.8 \% \\
(0.9 \%)\end{array}$ & $\begin{array}{l}12.3 \% \\
(0.9 \%)\end{array}$ & $\begin{array}{l}11.8 \% \\
(0.9 \%)\end{array}$ & $\begin{array}{l}11.3 \% \\
(0.9 \%)\end{array}$ & $\begin{array}{l}10.7 \% \\
(0.8 \%)\end{array}$ & $\begin{array}{l}-9.1 \% \\
(0.8 \%)\end{array}$ \\
\hline Sharpe Ratio & $\begin{array}{c}0.11 \\
(0.06)\end{array}$ & $\begin{array}{c}0.11 \\
(0.07)\end{array}$ & $\begin{array}{c}0.13 \\
(0.06)\end{array}$ & $\begin{array}{c}0.13 \\
(0.07)\end{array}$ & $\begin{array}{c}0.13 \\
(0.07)\end{array}$ & $\begin{array}{c}0.14 \\
(0.07)\end{array}$ & $\begin{array}{c}0.14 \\
(0.07)\end{array}$ & $\begin{array}{c}0.14 \\
(0.07)\end{array}$ & $\begin{array}{c}0.15 \\
(0.08)\end{array}$ & $\begin{array}{c}0.15 \\
(0.08)\end{array}$ & $\begin{array}{c}0.04 \\
(0.04)\end{array}$ \\
\hline \multicolumn{12}{|c|}{ Long Run Global Factor Prices: } \\
\hline Excess Returns & $\begin{array}{c}0.40 \% \\
(0.75 \%)\end{array}$ & $\begin{array}{c}0.26 \% \\
(0.70 \%)\end{array}$ & $\begin{array}{c}0.62 \% \\
(0.59 \%)\end{array}$ & $\begin{array}{c}0.67 \% \\
(0.56 \%)\end{array}$ & $\begin{array}{c}0.72 \% \\
(0.53 \%)\end{array}$ & $\begin{array}{c}0.75 \% \\
(0.51 \%)\end{array}$ & $\begin{array}{c}0.73 \% \\
(0.49 \%)\end{array}$ & $\begin{array}{c}0.73 \% \\
(0.47 \%)\end{array}$ & $\begin{array}{c}0.80 \% \\
(0.45 \%)\end{array}$ & $\begin{array}{c}0.90 \% \\
(0.43 \%)\end{array}$ & $\begin{array}{c}0.49 \% \\
(0.46 \%)\end{array}$ \\
\hline Excess Log Returns & $\begin{array}{c}-1.33 \% \\
(0.75 \%)\end{array}$ & $\begin{array}{c}-0.97 \% \\
(0.73 \%)\end{array}$ & $\begin{array}{c}-0.49 \% \\
(0.60 \%)\end{array}$ & $\begin{array}{l}-0.28 \% \\
(0.57 \%)\end{array}$ & $\begin{array}{c}-0.15 \% \\
(0.54 \%)\end{array}$ & $\begin{array}{c}-0.06 \% \\
(0.52 \%)\end{array}$ & $\begin{array}{c}-0.01 \% \\
(0.50 \%)\end{array}$ & $\begin{array}{c}0.04 \% \\
(0.49 \%)\end{array}$ & $\begin{array}{c}0.16 \% \\
(0.46 \%)\end{array}$ & $\begin{array}{c}0.32 \% \\
(0.44 \%)\end{array}$ & $\begin{array}{c}1.65 \% \\
(0.44 \%)\end{array}$ \\
\hline Sharpe Ratio & $\begin{array}{c}0.02 \\
(0.04)\end{array}$ & $\begin{array}{c}0.02 \\
(0.04)\end{array}$ & $\begin{array}{c}0.04 \\
(0.04)\end{array}$ & $\begin{array}{c}0.05 \\
(0.04)\end{array}$ & $\begin{array}{c}0.05 \\
(0.04)\end{array}$ & $\begin{array}{c}0.06 \\
(0.04)\end{array}$ & $\begin{array}{c}0.06 \\
(0.04)\end{array}$ & $\begin{array}{c}0.06 \\
(0.04)\end{array}$ & $\begin{array}{c}0.07 \\
(0.04)\end{array}$ & $\begin{array}{c}0.08 \\
(0.04)\end{array}$ & $\begin{array}{c}0.06 \\
(0.02)\end{array}$ \\
\hline \multicolumn{12}{|c|}{ Long Run Global Factor Prices, No Alpha: } \\
\hline Excess Returns & $\begin{array}{c}0.51 \% \\
(0.32 \%)\end{array}$ & $\begin{array}{c}0.55 \% \\
(0.29 \%)\end{array}$ & $\begin{array}{c}0.53 \% \\
(0.28 \%)\end{array}$ & $\begin{array}{c}0.49 \% \\
(0.27 \%)\end{array}$ & $\begin{array}{c}0.48 \% \\
(0.27 \%)\end{array}$ & $\begin{array}{c}0.48 \% \\
(0.26 \%)\end{array}$ & $\begin{array}{c}0.48 \% \\
(0.26 \%)\end{array}$ & $\begin{array}{c}0.47 \% \\
(0.25 \%)\end{array}$ & $\begin{array}{c}0.47 \% \\
(0.25 \%)\end{array}$ & $\begin{array}{c}0.46 \% \\
(0.24 \%)\end{array}$ & $\begin{array}{l}-0.04 \% \\
(0.13 \%)\end{array}$ \\
\hline Excess Log Returns & $\begin{array}{c}-1.23 \% \\
(0.35 \%)\end{array}$ & $\begin{array}{c}-0.67 \% \\
(0.32 \%)\end{array}$ & $\begin{array}{c}-0.58 \% \\
(0.30 \%)\end{array}$ & $\begin{array}{c}-0.47 \% \\
(0.29 \%)\end{array}$ & $\begin{array}{c}-0.40 \% \\
(0.29 \%)\end{array}$ & $\begin{array}{c}-0.33 \% \\
(0.28 \%)\end{array}$ & $\begin{array}{c}-0.27 \% \\
(0.28 \%)\end{array}$ & $\begin{array}{c}-0.22 \% \\
(0.27 \%)\end{array}$ & $\begin{array}{c}-0.17 \% \\
(0.27 \%)\end{array}$ & $\begin{array}{c}-0.12 \% \\
(0.26 \%)\end{array}$ & $\begin{array}{c}1.11 \% \\
(0.15 \%)\end{array}$ \\
\hline Sharpe Ratio & $\begin{array}{c}0.03 \\
(0.02)\end{array}$ & $\begin{array}{c}0.03 \\
(0.02)\end{array}$ & $\begin{array}{c}0.03 \\
(0.02)\end{array}$ & $\begin{array}{c}0.03 \\
(0.02)\end{array}$ & $\begin{array}{c}0.04 \\
(0.02)\end{array}$ & $\begin{array}{c}0.04 \\
(0.02)\end{array}$ & $\begin{array}{c}0.04 \\
(0.02)\end{array}$ & $\begin{array}{c}0.04 \\
(0.02)\end{array}$ & $\begin{array}{c}0.04 \\
(0.02)\end{array}$ & $\begin{array}{c}0.04 \\
(0.02)\end{array}$ & $\begin{array}{c}0.02 \\
(0.01)\end{array}$ \\
\hline
\end{tabular}


Table A9: Decomposition of Inequality Growth (Change in the Variance of Log Account Value), Excluding IPO Investors

This table presents the same cross-sectional variance decomposition as in Table 4, but excludes the population of accounts which, in at least one month-end, are at least $80 \%$ invested in stocks within three-months of their IPO. The excluded "IPO investor" accounts represent about 27.5\% of our sample of accounts. The size deciles used as a basis for determining expected returns are based on the full-sample size decile cutoffs used in Table 4.
$\operatorname{Var}_{t}^{*}\left(v_{i, t+1}\right)-\operatorname{Var}_{t}^{*}\left(v_{i t}\right)$
Realized Change in Log Account Value Variance
0.0223

Panel A: Overall Decomposition

$\begin{array}{ll}\operatorname{Var}_{t}^{*}\left(r_{i, t+1}\right)+2 \operatorname{Cov}_{t}^{*}\left(v_{i t}, r_{i, t+1}\right) & \text { Returns } \\ \operatorname{Var}_{t}^{*}\left(f_{i, t+1}\right)+2 \operatorname{Cov}_{t}^{*}\left(v_{i t}, f_{i, t+1}\right) & \text { Net Flows } \\ 2 \operatorname{Cov}_{t}^{*}\left(r_{i, t+1}, f_{i, t+1}\right) & \text { Covariance of Returns and Flows } \\ x_{i, t+1} & \text { Account Entry and Exit }\end{array}$

Panel B: Returns Component (Panel A, item [I])

$$
\operatorname{Var}_{t}^{*}\left(r_{i, t+1}\right)
$$

$2 \operatorname{Cov}_{t}^{*}\left(v_{i t}, r_{i, t+1}\right)$

Average Share of Change in Log Account Value Variance

Panel C: Account Value and Returns Covariance Component (Panel B, item [B])

$$
2 \operatorname{Cov}_{t}^{*}\left(b_{i t}, r_{i, t+1}\right)
$$

$2 \operatorname{Cov}_{t}^{*}\left(v_{i t}-b_{i t}, r_{i, t+1}\right)$

[I] $64.7 \%$

[II] $\quad 76.2 \%$

[III] $\quad-17.8 \%$

[IV] $-23.1 \%$

Panel D: Account Value and Returns Covariance Component (Panel B, item [B])

\section{$2 \operatorname{Cov}_{t}^{*}\left(v_{i t}, \ln \left(\overline{R_{i t}}\right)\right)$}

$2 \operatorname{Cov}_{t}^{*}\left(v_{i t}, r_{i, t+1}-\ln \left(\overline{R_{i t}}\right)\right)$

Panel E: Returns Component (Panel A, item [I])

$\operatorname{Var}_{t}^{*}\left(\mu_{i t}\right)$
$\operatorname{Var}_{t}^{*}\left(\varepsilon_{i, t+1}\right)$
$2 \operatorname{Cov}_{t}^{*}\left(\mu_{i t}, \varepsilon_{i, t+1}\right)$
$2 \operatorname{Cov}_{t}^{*}\left(v_{i t}, \mu_{i t}\right)$
$2 \operatorname{Cov}_{t}^{*}\left(v_{i t}, \varepsilon_{i, t+1}\right)$

$\begin{array}{ll}\text { [A] } & 57.7 \% \\ \text { [B] } & 42.3 \%\end{array}$

0.0144

$\begin{array}{ll}\text { [A] } & 57.7 \% \\ \text { [B] } & 42.3 \%\end{array}$

[1] 0.0061

$\begin{array}{ll}{[1]} & 74.0 \% \\ {[2]} & 26.0 \%\end{array}$

[a] 0.0061

[b] $\quad-161.1 \%$

Model 2

Model 1 Model 2

Mean Realized Return $\quad$ Long-Run Global Factor Long-Run Global Factor

$\begin{array}{cccc} & & \text { Prices } & \text { Prices, No Alph } \\ \text { [i] } & 0.0 \% & 0.2 \% & 0.1 \% \\ \text { [ii] } & 57.7 \% & 57.8 \% & 57.7 \% \\ \text { [iii] } & 0.0 \% & -0.2 \% & -0.2 \% \\ \text { [iv] } & 38.2 \% & 127.6 \% & 109.7 \% \\ \text { [v] } & 4.1 \% & -85.3 \% & -67.4 \%\end{array}$


Table A10: Decomposition of Inequality Growth (Change in the Variance of Log Account Value), Excluding First Year of Investing This table presents the same cross-sectional variance decomposition as in Table 4, but excludes the first 12 months of each account's equity investing history. The size deciles used as a basis for determining expected returns are based on the full-sample size decile cutoffs used in Table 4.
$\operatorname{Var}_{t}^{*}\left(v_{i, t+1}\right)-\operatorname{Var}_{t}^{*}\left(v_{i t}\right)$
Realized Change in Log Account Value Variance
0.0203

Panel A: Overall Decomposition

$\operatorname{Var}_{t}^{*}\left(r_{i, t+1}\right)+2 \operatorname{Cov}_{t}^{*}\left(v_{i t}, r_{i, t+1}\right)$
$\operatorname{Var}_{t}^{*}\left(f_{i, t+1}\right)+2 \operatorname{Cov}_{t}^{*}\left(v_{i t}, f_{i, t+1}\right)$
$2 \operatorname{Cov}_{t}^{*}\left(r_{i, t+1}, f_{i, t+1}\right)$
$x_{i, t+1}$

Returns

[I]

Average Share of Change in Log Account Value Variance

$x_{i, t+1}$

Net Flows

Covariance of Returns and Flows

Account Entry and Exit

$\begin{array}{cc}{[\mathrm{I}]} & 77.3 \% \\ \text { [II] } & 135.2 \% \\ \text { [III] } & -20.3 \% \\ {[\mathrm{IV}]} & -92.1 \%\end{array}$

Panel B: Returns Component (Panel A, item [I]) $\operatorname{Var}_{t}^{*}\left(r_{i, t+1}\right)$
$2 \operatorname{Cov}_{t}^{*}\left(v_{i t}, r_{i, t+1}\right)$

[A]

Panel C: Account Value and Returns Covariance Component (Panel B, item [B])

$$
2 \operatorname{Cov}_{t}^{*}\left(b_{i t}, r_{i, t+1}\right)
$$

$2 \operatorname{Cov}_{t}^{*}\left(v_{i t}-b_{i t}, r_{i, t+1}\right)$

[B]

0.0156

Panel D: Account Value and Returns Covariance Component (Panel B, item [B])

$$
2 \operatorname{Cov}_{t}^{*}\left(v_{i t}, \ln \left(\overline{R_{i t}}\right)\right)
$$

$2 \operatorname{Cov}_{t}^{*}\left(v_{i t}, r_{i, t+1}-\ln \left(\overline{R_{i t}}\right)\right)$

Panel E: Returns Component (Panel A, item [I])

$$
\begin{aligned}
& \operatorname{Var}_{t}^{*}\left(\mu_{i t}\right) \\
& \operatorname{Var}_{t}^{*}\left(\varepsilon_{i, t+1}\right) \\
& 2 \operatorname{Cov}_{t}^{*}\left(\mu_{i t}, \varepsilon_{i, t+1}\right) \\
& 2 \operatorname{Cov}_{t}^{*}\left(v_{i t}, \mu_{i t}\right) \\
& 2 \operatorname{Cov}_{t}^{*}\left(v_{i t}, \varepsilon_{i, t+1}\right)
\end{aligned}
$$

[B]

$45.6 \%$

[1] $\quad 0.0071$

[2] 29.8\%

[a] $-123.1 \%$

0.0071

[b] 223.1\%

0.0156

Model $1 \quad$ Model 2

Mean Realized Return $\quad$ Long-Run Global Factor $\quad$ Long-Run Global Factor

$\begin{array}{lccc}\text { [i] } & 0.0 \% & 0.2 \% & 0.1 \% \\ \text { [ii] } & 54.4 \% & 54.5 \% & 54.4 \% \\ \text { [iii] } & 0.0 \% & -0.3 \% & -0.2 \% \\ \text { [iv] } & 43.2 \% & 133.4 \% & 99.9 \% \\ \text { [v] } & 2.4 \% & -87.8 \% & -54.3 \%\end{array}$


Table A11: Decomposition of Inequality Growth (Change in the Variance of Log Account Value), Excluding Exiting Accounts

This table presents the same cross-sectional variance decomposition as in Table 4, but excludes the $49 \%$ of accounts that exit the stock market for more than one

consecutive month at some point during our sample period. The size deciles used as a basis for determining expected returns are based on the full-sample size decile cutoffs used in Table 4.

$\operatorname{Var}_{t}^{*}\left(v_{i, t+1}\right)-\operatorname{Var}_{t}^{*}\left(v_{i t}\right) \quad$ Realized Change in Log Account Value Variance

Panel A: Overall Decomposition

$\operatorname{Var}_{t}^{*}\left(r_{i, t+1}\right)+2 \operatorname{Cov}_{t}^{*}\left(v_{i t}, r_{i, t+1}\right)$

$\operatorname{Var}_{t}^{*}\left(f_{i, t+1}\right)+2 \operatorname{Cov}_{t}^{*}\left(v_{i t}, f_{i, t+1}\right)$

$2 \operatorname{Cov}_{t}^{*}\left(r_{i, t+1}, f_{i, t+1}\right)$

$x_{i, t+1}$

Returns

Returns

Net Flows

Covariance of Returns and Flows

Account Entry and Exit
0.0192

Average Share of Change in Log Account Value Variance

Panel B: Returns Component (Panel A, item [I])

$$
\operatorname{Var}_{t}^{*}\left(r_{i, t+1}\right)
$$$$
2 \operatorname{Cov}_{t}^{*}\left(v_{i t}, r_{i, t+1}\right)
$$

Panel C: Account Value and Returns Covariance Component (Panel B, item [B])

$$
\begin{aligned}
& 2 \operatorname{Cov}_{t}^{*}\left(b_{i t}, r_{i, t+1}\right) \\
& 2 \operatorname{Cov}_{t}^{*}\left(v_{i t}-b_{i t}, r_{i, t+1}\right)
\end{aligned}
$$

Panel D: Account Value and Returns Covariance Component (Panel B, item [B])

$$
2 \operatorname{Cov}_{t}^{*}\left(v_{i t}, \ln \left(\overline{R_{i t}}\right)\right)
$$$$
2 \operatorname{Cov}_{t}^{*}\left(v_{i t}, r_{i, t+1}-\ln \left(\overline{R_{i t}}\right)\right)
$$

Panel E: Returns Component (Panel A, item [I])

$$
\begin{aligned}
& \operatorname{Var}_{t}^{*}\left(\mu_{i t}\right) \\
& \operatorname{Var}_{t}^{*}\left(\varepsilon_{i, t+1}\right) \\
& 2 \operatorname{Cov}_{t}^{*}\left(\mu_{i t}, \varepsilon_{i, t+1}\right) \\
& 2 \operatorname{Cov}_{t}^{*}\left(v_{i t}, \mu_{i t}\right) \\
& 2 \operatorname{Cov}_{t}^{*}\left(v_{i t}, \varepsilon_{i, t+1}\right)
\end{aligned}
$$

[I]

[III]

[IV]

0.017

0.0095

[2] $24.3 \%$

0.0095

$\begin{array}{ll}\text { [a] } & -66.2 \% \\ \text { [b] } & 166.2 \%\end{array}$

Mean Realized Return ～Long-Run Global Factor Long-Run Global Factor

$\begin{array}{cccc}\text { [i] } & & \text { Prices } & \text { Prices, No A } \\ \text { [ii] } & 0.0 \% & 0.2 \% & 0.1 \% \\ \text { [iii] } & 45.9 \% & 45.9 \% & 45.9 \% \\ \text { [iv] } & 0.0 \% & -0.2 \% & -0.1 \% \\ \text { [v] } & 50.4 \% & 127.4 \% & 82.1 \% \\ & 3.7 \% & -73.3 \% & -28.0 \%\end{array}$

[B] $\quad 45.9 \%$

0.0176


Table A12: Alternative Decomposition of Inequality Growth (Change in the Variance of Log Account Value)

The first three components of the decomposition in Table 4 (returns, net flows, and their covariance), are combined here as the contribution of changing values of pre-existing accounts where returns are available. The remainder of the change in variance of log account value is then decomposed into the contribution of (pre-existing) accounts without returns data and the contribution of accounts that enter or exit the universe of stock holding accounts.

Average Share of Change in

Component Log Account Value Variance

Changing Value of Pre-existing Accounts with Returns (Returns, Flows)

0.0199

$101.30 \%$

Entry/Exit Due to Availability of Stock Return Data

$-0.0080$

$-40.91 \%$

Entry/Exit of Account from Stock Holding Population

0.0078

$39.61 \%$

Realized Change in Log Account Value Variance

0.0197

$100.00 \%$ 